\title{
The discovery of WASP-151b, WASP-153b, WASP-156b: Insights on giant planet migration and the upper boundary of the Neptunian desert ${ }^{\star}$
}

\author{
O. D. S. Demangeon ${ }^{1}$, F. Faedi ${ }^{2,9}$, G. Hébrard ${ }^{3,4}$, D. J. A. Brown ${ }^{2}$, S. C. C. Barros ${ }^{1}$, A. P. Doyle ${ }^{2}$,
} P. F. L. Maxted ${ }^{12}$, A. Collier Cameron ${ }^{7}$, K. L. Hay ${ }^{7}$, J. Alikakos ${ }^{18}$, D. R. Anderson ${ }^{12}$, D. J. Armstrong ${ }^{2,6}$, P. Boumis ${ }^{18}$, A. S. Bonomo ${ }^{8}$, F. Bouchy ${ }^{5}$, L. Delrez ${ }^{21,10}$, M. Gillon ${ }^{21}$, C. A. Haswell ${ }^{17}$, C. Hellier ${ }^{12}$, E. Jehin ${ }^{21}$, F. Kiefer ${ }^{3}$, K. W. F. Lam ${ }^{2}$, M. Lendl ${ }^{20,5}$, L. Mancini ${ }^{19,16,8}$, J. McCormac ${ }^{2}$, A. J. Norton ${ }^{17}$, H. P. Osborn ${ }^{2}$, E. Palle ${ }^{14,15}$, F. Pepe ${ }^{5}$, D. L. Pollacco ${ }^{2}$, J. Prieto-Arranz ${ }^{14,15}$, D. Queloz ${ }^{10,5}$, D. Ségransan ${ }^{5}$, B. Smalley ${ }^{12}$, A. H. M. J. Triaud ${ }^{11,13}$, S. Udry ${ }^{5}$, R. West ${ }^{2}$, and P. J. Wheatley ${ }^{2}$

1 Instituto de Astrofísica e Ciências do Espaço, Universidade do Porto, CAUP, Rua das Estrelas, 4150-762 Porto, Portugal e-mail: olivier.demangeon@astro.up.pt

2 University of Warwick, Department of Physics, Gibbet Hill Road, Coventry CV4 7AL, UK

3 Institut d'Astrophysique de Paris, UMR7095 CNRS, Université Pierre \& Marie Curie, 98bis boulevard Arago, 75014 Paris, France

4 Observatoire de Haute-Provence, Université d'Aix-Marseille \& CNRS, 04870 Saint Michel l'Observatoire, France

5 Observatoire de Genève, Université de Genève, 51 Chemin des Maillettes, 1290 Sauverny, Switzerland

6 Astrophysics Research Centre, Queen's University Belfast, University Road, Belfast BT7 1NN, UK

7 Centre for Exoplanet Science, SUPA, School of Physics and Astronomy, University of St. Andrews, St. Andrews KY16 9SS, UK

8 INAF-Osservatorio Astrofisico di Torino, via Osservatorio 20, 10025 Pino Torinese, Italy

9 INAF-Osservatorio Astrofisico di Catania, via S. Sofia 78, 95123 Catania, Italy

10 Cavendish Laboratory, JJ Thompson Avenue, CB3 0HE Cambridge, UK

11 Institute of Astronomy, Madingley Road, CB3 OHA Cambridge, UK

12 Astrophysics Group, Keele University, Staffordshire ST5 5BG, UK

13 School of Physics \& Astronomy, University of Birmingham, Edgbaston, Birmingham B15 2TT, UK

14 Instituto de Astrosfísica de Canarias (IAC), 38205 La Laguna, Tenerife, Spain

15 Departamento de Astrofísica, Universidad de La Laguna (ULL), 38206 La laguna, Tenerife, Spain

16 Max Planck Institue for Astronomy, Königstuhl 17, 69117 Heidelberg, Germany

17 School of Physical Sciences, The Open University, Milton Keynes MK7 6 AA, UK

18 Institute for Astronomy, Astrophysics, Space Applications and Remote Sensing, National Observatory of Athens, 15236 Penteli, Greece

19 Department of Physics, University of Rome Tor Vergata, via della Ricerca Scientifica 1, 00133 Roma, Italy

20 Space Research Institute, Austrian Academy of Sciences, Schmiedlstr. 6, 8042 Graz, Austria

21 Space sciences, Technologies and Astrophysics Research (STAR) Institute, Université de Liège, Allée du 6 Août 17, Bat. B5C, 4000 Liège, Belgium

Received 7 August 2017 / Accepted 16 October 2017

\section{ABSTRACT}

To investigate the origin of the features discovered in the exoplanet population, the knowledge of exoplanets' mass and radius with a good precision $(\lesssim 10 \%)$ is essential. To achieve this purpose the discovery of transiting exoplanets around bright stars is of prime interest. In this paper, we report the discovery of three transiting exoplanets by the SuperWASP survey and the SOPHIE spectrograph with mass and radius determined with a precision better than 15\%. WASP-151b and WASP-153b are two hot Saturns with masses, radii, densities and equilibrium temperatures of $0.31_{-0.03}^{+0.04} M_{\mathrm{J}}, 1.13_{-0.03}^{+0.03} R_{\mathrm{J}}, 0.22_{-0.02}^{+0.03} \rho_{\mathrm{J}}$ and $1290_{-10}^{+20} \mathrm{~K}$, and $0.39_{-0.02}^{+0.02} M_{\mathrm{J}}, 1.55_{-0.08}^{+0.10} R_{\mathrm{J}}$, $0.11_{-0.02}^{+0.02} \rho_{\mathrm{J}}$ and $1700_{-40}^{+40} \mathrm{~K}$, respectively. Their host stars are early $\mathrm{G}$ type stars (with mag $V \sim 13$ ) and their orbital periods are 4.53 and 3.33 days, respectively. WASP-156b is a super-Neptune orbiting a $\mathrm{K}$ type $\operatorname{star}$ (mag $V=11.6$ ). It has a mass of $0.128_{-0.009}^{+0.010} M_{\mathrm{J}}$, a radius of $0.51_{-0.02}^{+0.02} R_{\mathrm{J}}$, a density of $1.0_{-0.1}^{+0.1} \rho_{\mathrm{J}}$, an equilibrium temperature of $970_{-20}^{+30} \mathrm{~K}$ and an orbital period of 3.83 days. The radius of WASP$151 \mathrm{~b}$ appears to be only slightly inflated, while WASP-153b presents a significant radius anomaly compared to a recently published model. WASP-156b, being one of the few well characterized super-Neptunes, will help to constrain the still debated formation of Neptune size planets and the transition between gas and ice giants. The estimates of the age of these three stars confirms an already observed tendency for some stars to have gyrochronological ages significantly lower than their isochronal ages. We propose that high eccentricity migration could partially explain this behavior for stars hosting a short period planet. Finally, these three planets also lie close to (WASP-151b and WASP-153b) or below (WASP-156b) the upper boundary of the Neptunian desert. Their characteristics support that the ultra-violet irradiation plays an important role in this depletion of planets observed in the exoplanet population.

Key words. planets and satellites: detection - techniques: radial velocities - techniques: photometric stars: individual: WASP-151 - stars: individual: WASP-153 - stars: individual: WASP-156

\footnotetext{
* The radial velocity (Tables A.1-A.3) and the high resolution photometric data are only available at the CDS via anonymous ftp to cdsarc.u-strasbg.fr (130.79.128.5) or via http://cdsarc.u-strasbg.fr/viz-bin/qcat?J/A+A/610/A63
} 


\section{Introduction}

The successful harvest of exoplanets (see for example exoplanet.eu, Schneider et al. 2011) during the last two decades completely metamorphosed the field of exoplanet science. The initial assumption that the solar system was a typical example of planetary systems is long gone (as stated by Mayor \& Queloz 2012). The Kepler mission (Borucki et al. 2010) delivered 4496 transiting planetary candidates, including 2248 confirmed planets (according to the NASA Exoplanet Archive ${ }^{1}$, August 2017). This sample revealed various features of the exoplanet population demonstrating the necessity of a very large sample to encompass the exoplanets' diversity (see Borucki 2017, for a recent review). One of many surprising results from Kepler is that the orbital distance of exoplanets appears to be nearly random regardless of their size (e.g., Fabrycky et al. 2014). One striking exception to this observation is the so called subjovian desert or short period Neptunian desert (e.g. Mazeh et al. 2016; Matsakos \& Königl 2016; Kurokawa \& Nakamoto 2014; Szabó \& Kiss 2011). It corresponds to a depletion of planets at short orbital periods $(P<10$ days $)$ with masses or radius between super-Earth and sub-jovian planets (see Fig. 10). One possible explanation for this desert is the strong irradiation (bolometric and in particular extreme ultra-violet) from the parent star at those short orbital distances, especially at the early stages of the star's life. The strong stellar irradiation might have striped away the atmosphere of sub-jovian planets which had quickly migrated to the vicinity of their parent star and were not massive enough to retain their atmosphere, only leaving a super-Earth size core (e.g., Lundkvist et al. 2016; Lecavelier Des Etangs 2007). The mechanism responsible for the presence of giant planets in the vicinity of their parent star is still debated. However, the discovery by David et al. (2016) of a super-Neptune size planet orbiting close to a 5-10 Myr old star suggests that high eccentricity migration (e.g., Rasio \& Ford 1996; Fabrycky \& Tremaine 2007) is unlikely for this system (the tidal circularisation happening at longer timescales) and only leaves disk migration (e.g., Lin et al. 1996; Ward 1997) and in-situ formation as possible scenarios. Understanding the origin of the Neptunian desert could thus change our vision of gas and ice giant planet formation and evolution.

Unfortunately a large fraction of the planets discovered by Kepler surrounding the Neptunian desert do not have an accurate (precision $\lesssim 10 \%{ }^{2}$ ) determination of their mass and radius due to the faintness of their parent star. In this context ground based transit photometry surveys like SuperWASP (Pollacco et al. 2006), targeting bright stars, are essential contributors. In this paper, we present the WASP and SOPHIE discovery of two hot Saturns and one warm super-Neptune, with mass and radius measured with a precision better than $15 \%$, and discuss their impact on the formation and evolution theories of ice and gas giants. In Sect. 2, we describe the photometric and radial velocity observations acquired on the three systems. In Sect. 3, we present our analysis of the data with the resulting stellar and planetary parameters. Finally in Sect. 4, we discuss the nature and composition of these planets and their impact on planet formation and evolution theory with a focus on

\footnotetext{
1 http://exoplanetarchive.ipac.caltech.edu/

2 The exact precision required is difficult to assess, but a precision of 20 to $30 \%$ on the planetary density is usually required to be able to discriminate between the main families of planets (see for example Benz et al. 2013; Grasset et al. 2009). This corresponds to an uncertainty on the radius of roughly $10 \%$.
}

the migration of the hot giant planet population and the upper boundary of the Neptunian desert.

\section{Observations}

\subsection{Discovery: WASP}

The Wide Angle Search for Planets (WASP) operates two robotic telescope arrays, each consisting of eight Canon 200m, f/1.8 lenses with e2v $2048 \times 2048$, Peltier-cooled CCDs, giving a field of view of $7.8 \times 7.8$ degrees and a pixel scale of $13.7^{\prime \prime}$ (Pollacco et al. 2006). SuperWASP is located at the Roque de los Muchachos Observatory on La Palma (ORM - ING, Canary Islands, Spain), while WASP-South is located at the South African Astronomical Observatory (SAAO - Sutherland, South Africa). Each array observes up to eight pointings per night with a typical cadence of $8 \mathrm{~min}$ and an exposure time of $30 \mathrm{~s}$, with each pointing being followed for roughly five months per observing season. In January 2009, SuperWASP received a significant system upgrade that improved our control of red noise sources such as temperature-dependent focus changes (Barros et al. 2011; Faedi et al. 2011), leading to substantially improved data quality.

All WASP data are processed by the custom-built reduction pipeline described in Pollacco et al. (2006), producing one light curve per observing season and camera. These light curves are passed through the SysRem (Tamuz et al. 2005) and TFA (Kovács et al. 2005) de-trending algorithms to reduce the effect of known systematic signals, before a search for candidate transit signals is performed using a custom implementation of the Box Least-Squares algorithm (BLS; Kovács et al. 2002), as described in Collier Cameron et al. (2006, 2007). Once candidate planets have been identified, a series of multi-season, multi-camera analyses are carried out to confirm the detection and improve upon initial estimates of the candidates' physical and orbital parameters, which are derived from the WASP data in conjunction with publicly available catalogs (e.g., UCAC4, Zacharias et al. 2013; 2MASS, Skrutskie et al. 2006). These additional analyses are essential for rejection of false positives, and for identification of the best candidates. This process allowed to detect three transit planets that we will now introduce.

1SWASPJ231615.22+001824.5 (2MASS23161522+0018242), hereafter WASP-151, lies very close to the celestial equator and is thus visible to both WASP arrays. A total of 45945 data were obtained between 2008-06-12 and 2012-11-28, 16375 from SuperWASP and 29570 by WASP-South. A search for periodic modulation in the WASP light curves, such as might be caused by stellar activity or rotation, was carried out using the method of Maxted et al. (2011). No significant periodicity was identified, and we place an upper limit of 2 mmag on the amplitude of any modulation. During these observations a total of 195 transits were covered of which 27 were full or quasi-full events. The WASP data show a periodic reduction in stellar brightness of approximately $0.01 \mathrm{mag}$, with a period of roughly 4.5 days, a duration of approximately $3.7 \mathrm{~h}$, and a shape indicative of a planetary transit. The WASP thumbnails of WASP-151 show some contamination from a background galaxy about $20^{\prime \prime}$ from the target and thus within our first aperture. The galaxy is about 3 mag fainter in $V$ than our target. We calculated a dilution factor for WASP-151 of about $1 \%$ and thus negligible when considering WASP data.

1SWASPJ183702.97+400107.4 (2MASS18370297+4001073), hereafter WASP-153, is our second transiting planet host. 42349 photometric measurements were made by SuperWASP between 2004-05-14 and 2010-08-24, with no observations by 
Table 1. Summary of the photometric observation of WASP-151, WASP-153 and WASP-156.

\begin{tabular}{llll}
\hline \hline Date & Instrument & Filter & Comment \\
\hline \multicolumn{4}{c}{ WASP-151b } \\
\hline $06 / 2008 \rightarrow 11 / 2012$ & WASP & Johnson $R$ & detection \\
$03 / 09 / 2015$ & IAC80 & Johnson $R$ & full transit \\
$01 / 11 / 2015$ & IAC80 & Johnson $R$ & full transit \\
$15 / 06 / 2016$ & TRAPPIST & Sloan $z$ & full transit \\
$04 / 09 / 2016$ & EulerCam & NGTS & partial transit \\
$24 / 10 / 2016$ & EulerCam & NGTS & full transit \\
$12 / 2016 \rightarrow 03 / 2017$ & K2 & Kepler & 13 full transits \\
\hline \multicolumn{5}{c}{ WASP-153b } & \\
\hline $05 / 2004 \rightarrow 08 / 2010$ & WASP & Johnson $R$ & detection \\
$17 / 07 / 2015$ & Liverpool & Johnson $R$ & partial transit \\
$05 / 08 / 2017$ & RISE-2 & $V+R$ & full transit \\
\hline \multicolumn{4}{c}{ WASP-156b } \\
\hline $07 / 2008 \rightarrow 12 / 2010$ & WASP & Johnson $R$ & detection \\
$29 / 12 / 2014$ & EulerCam & Gunn $z$ & full transit \\
$07 / 11 / 2016$ & EulerCam & Gunn $r$ & full transit \\
$27 / 12 / 2016$ & NITES & Johnson $I$ & partial transit \\
\hline
\end{tabular}

WASP-South owing to the high declination of the target $\left(+40^{\circ}\right)$. We found no significant periodic modulation, and we place an upper limit of $1.5 \mathrm{mmag}$ on the amplitude of any such light curve variation. There are a total of 688 transits observed of which 54 are good events ${ }^{3}$. Our BLS searches identified the signature of a candidate transiting planet on a 3.3 days orbit, in the form of a periodic $0.006 \mathrm{mag}, 3 \mathrm{~h}$ reduction in stellar brightness.

1SWASPJ021107.61+022504.8 (2MASS02110763+0225050), hereafter WASP-156, is our third and last transiting planet host. We again found no significant periodic modulation, and we place an upper limit of $1 \mathrm{mmag}$ on the amplitude of any such light curve variation. As with WASP-151, the equatorial declination of WASP-156 allows both WASP arrays to monitor the star for flux variations. 22809 flux measurements were made, 13481 by SuperWASP and 9328 by WASP-South. A total of 230 transits were observed of which 23 are good events ${ }^{2}$. A $2.3 \mathrm{~h}$ long, $0.007 \mathrm{mag}$ reduction in brightness was found to repeat on a 3.8 days period with a typical planetary transit-like shape.

\subsection{Photometric follow-up}

\subsubsection{Ground-based photometric follow-up observations}

The WASP consortium has access to multiple observing facilities that can be used to obtain additional in-transit photometric observations. These follow-up light curves are used to confirm the presence of the candidate signal, particularly useful in the case of unreliable initial ephemerides, and are also used to improve the accuracy of our light-curve modeling, and to constrain the system parameters more precisely. A list of the follow-up photometric observations for our three planets is presented in Table 1.

\footnotetext{
3 Good events refers to full transit observations which did not suffer from obvious deformations due to the conditions of observation.
}

WASP-151b. Two full transits of WASP-151 were observed on 2015-09-03 and 2015-11-01 with the CAMELOT camera of the $0.82 \mathrm{~m}(f / 11.3)$ IAC-80 telescope, which is operated on the island of Tenerife by the Instituto de Astrofísica de Canarias (IAC) at the Spanish Observatorio del Teide. CAMELOT has a $2048 \times 2048$ pixel CCD with a scale of $0.304^{\prime \prime}$ pixel $^{-1}$ and a $10.6^{\prime}$ field-of-view. Images were bias and flat-field corrected using standard techniques.

An additional full transit was observed on 2016-06-15 with the robotic $0.6 \mathrm{~m}$ TRAnsiting Planets and PlanetesImals Small Telescope (TRAPPIST; Jehin et al. 2011; Gillon et al. 2011) at the La Silla Observatory operated by the European Southern Observatory (ESO). TRAPPIST is equipped with a thermoelectrically-cooled $2 \mathrm{~K} \times 2 \mathrm{~K} C \mathrm{CD}$ with a pixel scale of $0.65^{\prime \prime}$, giving a $22^{\prime} \times 22^{\prime}$ field of view. A Sloan- $z^{\prime}$ filter was used for the transit observations of this system, during which the positions of the stars on the chip were maintained to within a few pixels thanks to a software guiding system that regularly derives an astrometric solution for the most recently acquired image and sends pointing corrections to the mount if needed. After carrying out bias, dark, and flat-field corrections we extract stellar fluxes from our images using the IRAF ${ }^{4}$ DAOPHOT aperture photometry software (Stetson 1987). Several sets of reduction parameters were tested on stars of similar brightness to WASP-151, from which we selected the set giving the most precise photometry. After a careful selection of reference stars, the transit light curves were finally obtained using differential photometry.

The $1.2 \mathrm{~m}$ Swiss telescope using EulerCam (Lendl et al. 2012), also at La Silla, observed a full transit of WASP-151b on 2016-10-24 and a partial transit on 2016-10-24. In both cases, a filter with a central wavelength of $698 \mathrm{~nm}$ and an effective bandwidth of $312 \mathrm{~nm}$ was used; this filter is the same as that used by the Next Generation Transit Survey (NGTS; Wheatley et al. 2013, 2014). The Swiss telescope employs an absolute tracking system which matches point sources in each image with a catalog and adjusts the telescope's pointing between exposure to compensate for drift. In this manner, the pixel position of the star is maintained throughout. All data were reduced as outlined in Lendl et al. (2012), and light curves were produced through differential aperture photometry. To minimize scatter in the light curves, we carefully selected the most stable field stars to use as references.

WASP-153b. A partial transit of WASP-153b was observed on 2015-07-17 in the Johnson- $R$ filter using the RISE instrument mounted on the robotic Liverpool Telescope (LT; Steele et al. 2004) at ORM. RISE is equipped with a back-illuminated, frame-transfer, $1024 \times 1024$ pixel CCD. Images were automatically bias, dark, and flat-field corrected by the standard RISE reduction pipeline, which uses standard IRAF routines.

A full transit was later obtained with RISE- 2 mounted on the $2.3 \mathrm{~m}$ telescope situated at Helmos observatory in Greece on 5 August 2017. The CCD size is $1 \mathrm{~K} \times 1 \mathrm{~K}$ with a pixel scale of $0.51^{\prime \prime}$ and a field of view of $9^{\prime} \times 9^{\prime}$ (Boumis et al. 2010). The exposure time was $12 \mathrm{~s}$ and the $V+R$ filter was used. As for the previous transit observation, the images were processed standard RISE reduction pipeline.

4 IRAF is distributed by the National Optical Astronomy Observatories, which are operated by the Association of Universities for Research in Astronomy, Inc., under cooperative agreement with the National Science Foundation. 
WASP-156b A partial transit of WASP-156b was observed in the Johnson-I filter on 2016-12-27 using the Near Infra-red Transiting ExoplanetS (NITES) Telescope (McCormac et al. 2014), located at ORM. NITES is a semi-robotic, $0.4 \mathrm{~m}(f / 10)$ Meade LX200GPS Schmidt-Cassegrain telescope, mounted with a Finger Lakes Instrumentation Proline 4710 camera and a $1024 \times$ 1024 pixel deep-depleted CCD made by e2v. The telescope has a field of view of $11 \times 11^{\prime}$ squared, and a pixel scale of $0.66^{\prime \prime}$ pixel $^{-1}$. Autoguiding was performed using the DONUTS algorithm (McCormac et al. 2013). After performing bias and flat-field corrections using PyRAF ${ }^{5}$ and the standard routines in IRAF, aperture photometry was performed using DAOPHOT and multiple comparison stars, selected to minimize the RMS scatter in the out-of-transit light curve.

In addition to the NITES observations, EulerCam was used to observe two full transits of WASP-156b, on 2014-12-29 using a Gunn-z filter and on 2016-11-07 using a Gunn-r filter. The 2014 observations, however, are unreliable owing to large PSF variations, and stellar counts in the non-linear regime of the $E u$ lerCam CCD.

\subsubsection{K2 observations of WASP-151}

In addition to the ground-based photometric observations described in the previous sections, WASP-151 (alias EPIC 246441449) was observed by NASA's Kepler Space Telescope in its two-reaction wheel mission K2 (Howell et al. 2014) during Campaign 12. The observations span 79 days (from 15 December 2016 to 4 March 2017) except for the five days from 1 to 6 February 2017 when the spacecraft was in safe mode.

Since Campaign 9, the K2 consortium releases the raw cadence data shortly after downlink from the Kepler satellite. These data are raw, as opposed to the science cadence data like the target pixel files (TPF), for two main reasons ${ }^{6}$. First their format, the raw cadence data are provided as one file per cadence delivering the pixel counts for the whole focal plane as a table. In order to construct the image time series of a target, we need the pixel mapping reference file which specifies the (column, row) CCD coordinates for each value in the raw cadence data tables. Second, the raw cadence data are not calibrated. It means that they are not reduced with the Kepler pipeline (Quintana et al. 2010) and thus not corrected for background, dark, smearing trails, undershoot or non-linearity of the pixels response. The formatting and calibration of the raw cadence data for all the targets of a K2 campaign is a very lengthy procedure and even if the raw cadence data for Campaign 12 have been released several months ago, the calibrated TPF are, at this moment, still unavailable. Therefore, to be able to benefit from the high quality light curves of the WASP-151 system provided by the K2 mission, we decided to format and reduce ourselves the raw cadence data.

To obtain an image time series, we used the Kadenza ${ }^{7}$ software (Barentsen 2017) provided by the NASA's Kepler/K2 Guest Observer Office. Then, to extract the light curve, we used the Polar software (Barros et al. 2016) which performs a partial

\footnotetext{
PyRAF is a product of the Space Telescope Science Institute, which is operated by AURA for NASA.

6 For more details of the Kepler raw and science cadence data, we refer the reader to the technical note entitled Format Information for Cadence Pixel Files available at https://archive.stsci.edu/k2/ manuals/KADN-26315.pdf

7 The Kadenza software is available on GitHub at https://github. com/KeplerGO/kadenza or on Zenodo at https://doi.org/10. 5281/zenodo. 344973
}

calibration by subtracting the background and dark values thanks to estimates obtained on the images themselves. In parallel to the Polar reduction, we also reduced the image time series with the Python package Everest (Luger et al. 2016) to check the scientific validity of our reduction. Everest has been recently used to extract the light curve of the TRAPPIST-1 system observed by K2 during the same campaign (Luger et al. 2017) and thus in the same conditions. The two light curves are almost identical and compatible at 1 sigma giving us confidence in the scientific quality of our data reduction.

The light curve clearly displays transit features at the ephemeris inferred from the WASP data with no sign of outof-transit variations. A search for periodic modulation caused by stellar activity showed a tentative detection with an amplitude of $1 \mathrm{ppt}(\sim 1 \mathrm{mmag})$ at a period of 35 days. We then searched the light curve for additional transit features (apart from WASP151b's transit). We investigated a tentative mono transit-shaped feature which proved to be an artifact due to the position-flux decorrelation technique used by Polar. For this decorrelation, we cut the K2 image time series in several parts where the behavior of the pointing jitter of the Kepler satellite can be safely assumed to be 1 dimensional (for more details see Barros et al. 2016). The mono transit-shaped feature was appearing precisely at the junction of two of those parts. A slight change of the location of the cut made the feature disappear. Finally, no additional transit features was detected. For the analysis, we kept only intervals of twice the transit duration before and after each transit of WASP-151b. The phase-folded Polar-K2 light curve of WASP151 is shown in the bottom panel of Fig. 2.

\subsection{Spectroscopic follow-up}

The spectroscopic follow-up of these three candidates was mainly performed with SOPHIE, the spectrograph dedicated to high-precision radial velocity measurements at the $1.93-\mathrm{m}$ telescope of the Haute-Provence Observatory, France (Bouchy et al. 2009). For two systems, it was also complemented by radial velocities obtained with the CORALIE spectrograph at the 1.2-m Euler-Swiss telescope at La Silla (Queloz et al. 2000), Chile. The first goal of these spectroscopic observations is to establish the planetary nature of the transiting candidates found in photometry (see Sect. 2.3.2) The second goal is to characterize the secured planets by measuring in particular their masses and orbital eccentricities (see Sect. 3.2.1).

\subsubsection{Description of the observations}

SOPHIE was used in High-Efficiency mode with a resolving power $R=40000$ to increase the throughput for these faint stars. The exposure times ranged from 400 to $2200 \mathrm{~s}$ depending on the targets, and they were adjusted as a function of the weather conditions to keep the signal-to-noise ratio (S/N) as constant as possible for any given star. The spectra were extracted using the SOPHIE pipeline, and the radial velocities were measured from the weighted cross-correlation with numerical masks characteristic of the spectral type of the observed star (Baranne et al. 1996; Pepe et al. 2002). We adjusted the number of spectral orders used in the cross-correlation to reduce the dispersion of the measurements. Some spectral domains are noisy (especially in the blue part of the spectra) and using them would have degraded the accuracy of the radial-velocity measurement.

The error bars on the radial velocities were computed from the cross-correlation function using the method presented by 
Boisse et al. (2010). Some spectra were contaminated by moonlight. Following the method described in Pollacco et al. (2008) and Hébrard et al. (2008), we estimated and corrected for the moonlight contamination by using the second SOPHIE fiber aperture, which is targeted on the sky, while the first aperture points toward the star. This results in radial velocity corrections up to $40 \mathrm{~m} / \mathrm{s}$, and below $40 \mathrm{~m} / \mathrm{s}$ in most of the cases. Removing these points does not significantly modify the orbital solutions.

The CORALIE spectrograph has a resolution of $\sim 60000$. The observing strategy is made to ensure that observations are taken exclusively without Moon contamination and the second fiber is used to obtain a simultaneous calibration. Prior to April 2015 the calibration was done with a thorium-argon lamp, but since then it is done with a Fabry-Pérot unit. The reduction of the spectra and the production of the radial velocities proceed in a fashion very similar to the procedure applied to SOPHIE data. The radial velocity measurements are reported in Tables A.1A.3 (available at the CDS) and are displayed in Figs. 3, 5 and 7 together with their Keplerian fits and the residuals.

\subsubsection{Validation of the planetary nature}

The transit photometry method suffers from a high rate of false positives. Eclipsing binaries (EB), background eclipsing systems (BES) and hierarchical triple systems (HTS) can mimic the transit of a planet orbiting the target star and induce an erroneous identification of the nature and parameters of the transiting system (e.g., Díaz et al. 2014; Torres et al. 2011). Whenever it is possible, radial velocity measurements are used to rule out these false positive scenarios and validate the planetary nature of the transiting object. This validation is made in several steps.

1. The inspection of the spectra allows us to identify double lines spectrum which are a sign of spectroscopic binaries (SB2) or BES/HTS where the contaminating eclipsing system has a similar brightness than the target star.

2. Phase-folding the data at the period inferred from the transits allows us to estimate the amplitude of the RV signal at this period. Assuming that this amplitude is due to the reflex motion of the target star, it allows us to estimate the mass of the gravitationally bound companion and to identify single line binaries (SB1).

If those two steps are successfully passed, the EB scenario can be ruled out ${ }^{8}$. For our three planetary candidates, none of the measurements showed double lines. Furthermore, they showed variations in phase with the SuperWASP transit ephemeris and with semi-amplitudes between 20 and $40 \mathrm{~m} \mathrm{~s}^{-1}$, implying companion masses below 0.4 Jupiter mass. Therefore, we were able to exclude the EB hypothesis for our three cases. The remaining false positive scenarios are thus BES and HTS with faint ${ }^{9}$ contaminating eclipsing systems.

3. Extracting the radial velocities using masks corresponding to different spectral types allows us to identify some cases of

\footnotetext{
8 The following steps ( 3 and 4) rely on the fact that a significant RV variation is detected during the second step. If this is not the case, the only remaining solution is often to assess the nature of the transiting signal through probabilistic validation. There exist very few software programs able to perform this probabilistic validation: BLENDER (Torres et al. 2011), PASTIS (Díaz et al. 2014) and under more restrictive assumptions VESPA (Morton 2015, 2012).

9 As described in the first step, we can also exclude BES and HTS configurations involving bright contaminating eclipsing systems up to a flux ratio between the contaminant and the greater than $\sim 1 \%$.
}

HTS/BES where the star responsible for the RV signal has a different spectral type than the target star. In such a case, the RV amplitude will vary significantly with the mask used (e.g., Santos et al. 2002).

4. If the RV signal observed is due to a HTS/BES, it will display variation in the cross-correlation function bisector span (BS) correlated with the RV signal (Santerne et al. 2015). It is thus important to properly assess the correlation between $\mathrm{RV}$ and BS, since a significant correlation would exclude the planetary hypothesis ${ }^{10}$.

For our three planetary candidates, radial velocities were measured using different stellar masks (F0, G2, and K5) and produced variations with similar amplitudes. Furthermore, Fig. 1 shows the correlation diagram of the RV and BS signal along with the posterior probability density function of the correlation coefficient, obtained with the method and tools described in Figueira et al. (2016). The values and 95\% confidence intervals that we obtained are $0.19_{-0.31}^{+0.28},-0.01_{-0.26}^{+0.26}$ and $-0.13_{-0.24}^{+0.25}$ for WASP-151b, WASP-153b and WASP-156b respectively, meaning that no significant correlation is detected.

The final step that is rarely performed (a would-be step 5), when an RV variation is significantly detected, is to check whether or not a correlation could have been detected assuming that the RV variation is due to an HTS or a BES. Santerne et al. (2015) described in detail the expected RV and BS signals for HTS/BES. The exact degree of correlation and the exact amplitude ratio of $\mathrm{BS}$ over RV depend on the following factors: flux ratio, full width at half maximum (FWHM) of the crosscorrelation functions, mean radial velocity difference $(\phi)$ and spectral types. However in most configurations ${ }^{11}$, to be able to produce the $\sim 30 \mathrm{~m} \mathrm{~s}^{-1} \mathrm{RV}$ variation that we observe, the associated BS signal must have an amplitude equal to a significant fraction of the RV signal. This in turn implies that the ratio of the dispersion over the average error bar of the BS measurements $\left(\frac{\mathrm{std}(\mathrm{BS})}{\left\langle\sigma_{\mathrm{BS}}\right\rangle}\right)$ has to be greater than one. Consequently, we computed $\frac{\text { std(BS) }}{\left\langle\sigma_{\mathrm{BS}}\right\rangle}$ for our three stars and this ratio is compatible with one in all cases (see first column of Table 2). This implies that the dispersion of the BS values can be explained by the measurement uncertainties solely and discards cases where the additional BS signal due to the HTS/BES could have been detected. To quantify these cases, we computed the maximum fraction of the RV amplitude that the BS signal can have without producing a $2 \sigma$ departure from one of $\frac{\operatorname{std}(\mathrm{BS})}{\left\langle\sigma_{\mathrm{BS}}\right\rangle}$ (see second column of Table 2).

With Table 2, we can identify the configurations of HTS/BES that are excluded by our correlation and BS dispersion analyses, given the number and the precision of our RV and BS measurements. We thus conclude that for our three stars, we would have been able to detect the increase in the dispersion of the BS, and thus the correlation between RV and BS associated with the presence of most HTS/BES configurations. We are thus confident that the most likely explanation for our transits and RV signals is a planet orbiting the target stars.

\footnotetext{
${ }^{10}$ A correlation can be explained by a BES or a HTS but also by stellar activity (e.g., Queloz et al. 2001).

11 According to Santerne et al. (2015), the only HTS or BES configuration which might produce a RV signal with a comparatively low BS signal is when the FWHM or the target and the contaminating systems are similar and $\phi$ is low compared to this FWHM value. Given the FWHM of $\sim 5 \mathrm{~km} \mathrm{~s}^{-1}$ of our observation, this is only possible in a specific kind of HTS system.
} 

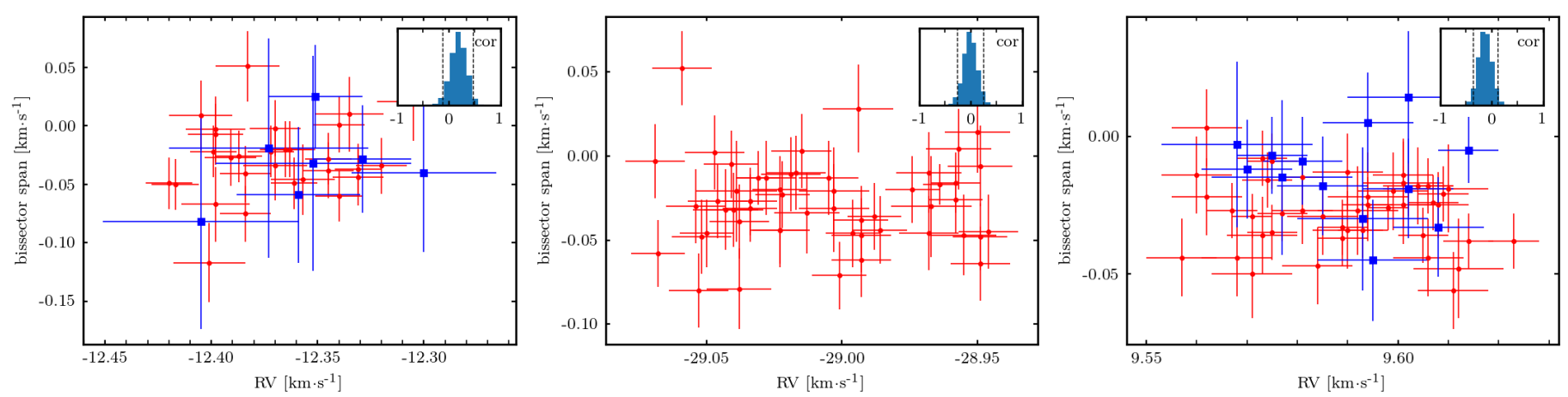

Fig. 1. Bisector span as a function of the radial velocities with $1 \sigma$ error bars for WASP-151, 153 and 156 (from left to right). SOPHIE data are the red circles; CORALIE data are the blue squares. The ranges here have the same extents in the $x$ - and $y$-axes. For each star, the posterior probability function of the correlation coefficient is displayed in an insert located in the upper left corner.

Table 2. Analysis of the dispersion of the bisector span.

\begin{tabular}{lcc}
\hline \hline Star & $\frac{\operatorname{std}(\mathrm{BS})}{\left\langle\sigma_{\mathrm{BS}}\right\rangle}$ & $\max \left(\frac{\mathrm{BS}}{\mathrm{RV}}\right)[\%]$ \\
\hline WASP-151 & $0.93 \pm 0.15$ & 84 \\
WASP-153 & $1.15 \pm 0.12$ & 22 \\
WASP-156 & $1.03 \pm 0.11$ & 48 \\
\hline
\end{tabular}

Notes. std(BS) indicates the standard deviation of the BS measurements. $\left\langle\sigma_{B S}\right\rangle$ indicates the average error bar on the individual BS measurements. $\operatorname{Max}\left(\frac{\mathrm{BS}}{\mathrm{RV}}\right)$ is the maximum fraction of the RV amplitude observed that the BS signal can have without producing a value of $\frac{\operatorname{std}(\mathrm{BS})}{\left\langle\sigma_{\mathrm{BS}}\right\rangle}$ which is significantly superior than one (see Sect. 2.3.2 for more details).

\section{Results}

\subsection{Stellar parameters from spectroscopy}

A total of 26, 46, and 40 individual SOPHIE spectra of WASP151, WASP-153 and WASP-156 were co-added to produce a single spectrum with a typical $\mathrm{S} / \mathrm{N}$ of around 50:1, 50:1 and 70:1, respectively. Here we used only the spectra without moonlight contamination; this enabled a sufficiently high $\mathrm{S} / \mathrm{N}$ to be reached with $R=40000$, and prevented any possible contamination in the spectra.

The standard pipeline reduction products were used in the analysis, which was performed using the methods given in Doyle et al. (2013). The effective temperature $\left(T_{\text {eff }}\right)$ was determined from the excitation balance of the Fe I lines. The ionisation balance of $\mathrm{Fe}$ I and Fe II was used as the surface gravity $(\log g)$ diagnostic. The metallicity $([\mathrm{Fe} / \mathrm{H}])$ was determined from equivalent width measurements of several unblended lines. They are more accurate and agree with the measurements secured from the cross-correlation function following Boisse et al. (2010). A value for microturbulence $\left(\xi_{\mathrm{t}}\right)$ was determined from Fe lines by requiring that there is no slope between the abundance and the equivalent width. The error estimates for $\xi_{\mathrm{t}}$ include the uncertainties in $T_{\text {eff }}$ and $\log g$, as well as the scatter due to the measurement and the atomic data uncertainties. Values for macroturbulence $\left(v_{\text {mac }}\right)$ were determined from the calibration of Doyle et al. (2014), however the value for WASP-156 is extrapolated from the calibration as this star is not within the correct temperature range. With the $v_{\text {mac }}$ fixed to the calibration value, the projected stellar rotation velocity $\left(v \sin i_{*}\right)$ was determined by fitting the profiles of several unblended lines.
Here again, the $v \sin i_{*}$ values agree with those obtained from the cross-correlation function following Boisse et al. (2010).

Lithium is detected in WASP-151 and WASP-153, with an equivalent width of $17 \mathrm{~m} \AA$ and $98 \mathrm{~m} \AA$, corresponding to an abundance $\log A(\mathrm{Li})$ of $1.73 \pm 0.05$ and $2.77 \pm 0.05$ respectively. This implies an age of several Gyr and several Myr respectively. There is no significant detection of lithium in WASP-156, with an equivalent width upper limit of $11 \mathrm{~m} \AA$, corresponding to an abundance upper limit of $\log A(\mathrm{Li})<0.2$. This implies an age of at least $500 \mathrm{Myr}$ (Sestito \& Randlich 2005).

The rotation rate $(P=14.8 \pm 4 \mathrm{~d})$ implied by the $v \sin i_{*}$ gives a gyrochronological age of $\sim 1.80_{-1.00}^{+2.03}$ Gyr using the Barnes (2007) relation for WASP-151. Similarly, the rotation rate of $P=$ $11.7 \pm 2 \mathrm{~d}$ gives an age of $\sim 1.21_{-0.60}^{+1.19} \mathrm{Gyr}$ for WASP-153, and the rotation rate of $P=12.6 \pm 4 \mathrm{~d}$ gives an age of $\sim 0.58_{-0.31}^{+0.51} \mathrm{Gyr}$ for WASP-156.

Finally from $T_{\text {eff }}, \log g$ and $[\mathrm{Fe} / \mathrm{H}]$, we inferred stellar mass and radius estimates using the Torres et al. (2010) calibration. The parameters and error bars obtained from this analysis are listed in the section stellar parameters of Table A.4.

\subsection{System parameters}

\subsubsection{Transit and RV analysis}

We followed the same method to perform inference of the parameters for the three systems. We jointly analysed all the radial velocity and photometric datasets available for a given system. To model the radial velocity and photometric data, we used the Python packages ajplanet ${ }^{12}$ (Espinoza et al. 2016) and batman $^{12}$ (Kreidberg 2015) respectively. In order to decrease the correlation between the parameters of our model and ease the fit, we adopted the parametrisation suggested by Eastman et al. (2013) with $R_{\mathrm{p}} / R_{*}$ the ratio of the planet's radius to that of the star, $P$ the orbital period, $t_{c}$ the planet's time of inferior conjunction, $\sqrt{e} \cos \omega_{*}$ and $\sqrt{e} \sin \omega_{*}$ where $e$ is the orbital eccentricity and $\omega_{*}$ is the stellar orbital argument of periastron, $K$ the radial velocity semi-amplitude, $i$ the orbital inclination, $a / R_{*}$ the ratio of the planet's orbital semi-major axis over the stellar radius, $v 0$ the systemic radial velocity, $u$ and $v$ the two coefficients of the limb-darkening quadratic law. To this set of parameters we added a logarithmic multiplicative jitter factor $\left(\ln f_{\sigma}\right)$ for each

12 Several of the Python packages used for this work are publicly available on Github: ajplanet at https://github.com/ andres-jordan/ajplanet, batman at https://github.com/ $1 \mathrm{kreidberg/batman,} \mathrm{emcee} \mathrm{at} \mathrm{https://github.com/dfm/emcee,}$ ldtk at https://github.com/hpparvi/ldtk 

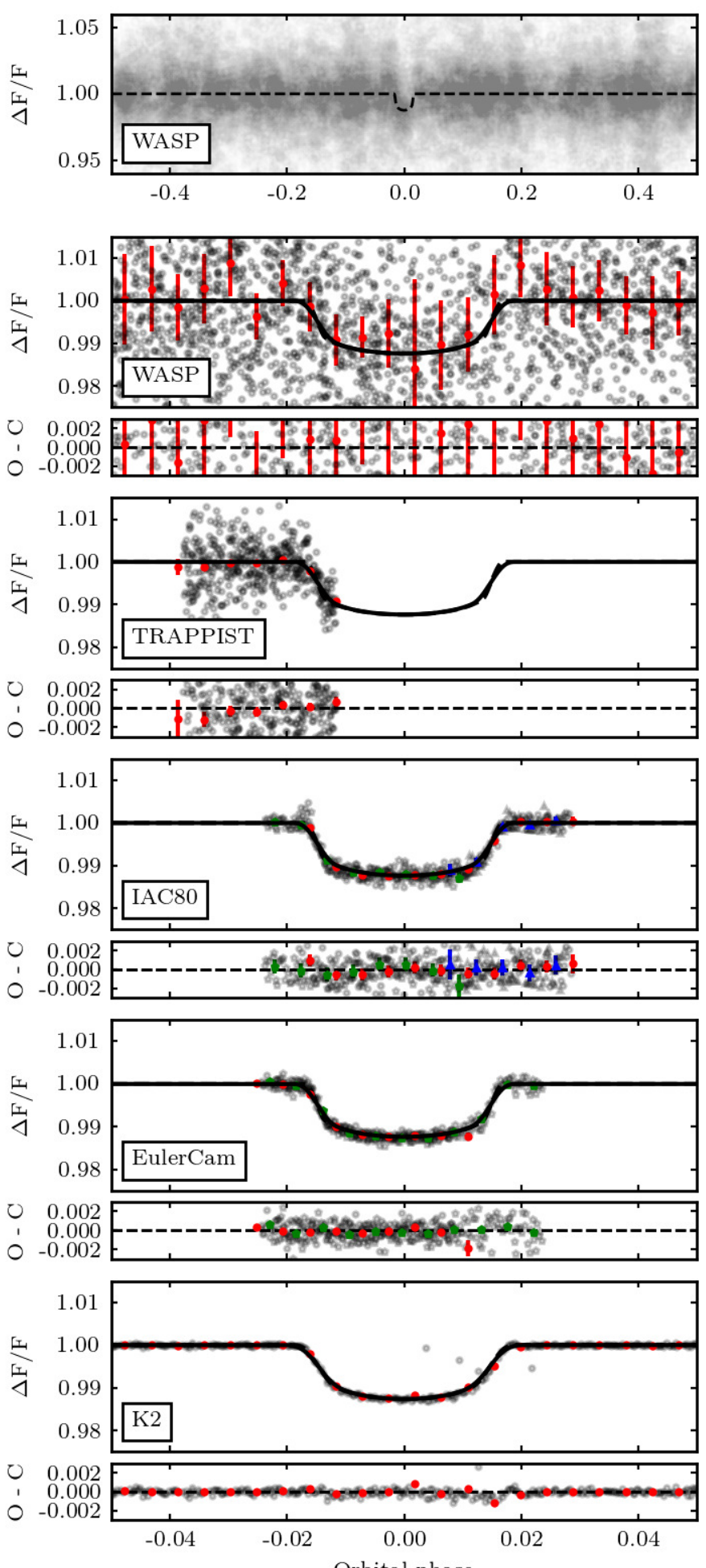

Orbital phase

Fig. 2. Photometry of WASP-151. The black or gray points are the data points at the original cadence of the observations, displayed without error bars for clarity. The red points corresponds to the same data points binned in phase with a bin width equivalent to $29.424 \mathrm{~min}$ (Kepler long cadence). These points are represented with their associated $1 \sigma$ error bars. The black dashed and solid lines correspond to the best-fit model at the original and binned cadence respectively. When several datasets have been gathered with the same instrument, they are displayed on the same figure but with different symbols and colors. September $E u$ lerCam and IAC80 data are red dots, October EulerCam and first part of the November IAC80 data are green pentagons, and second part of the November IAC 80 data are blue triangles.

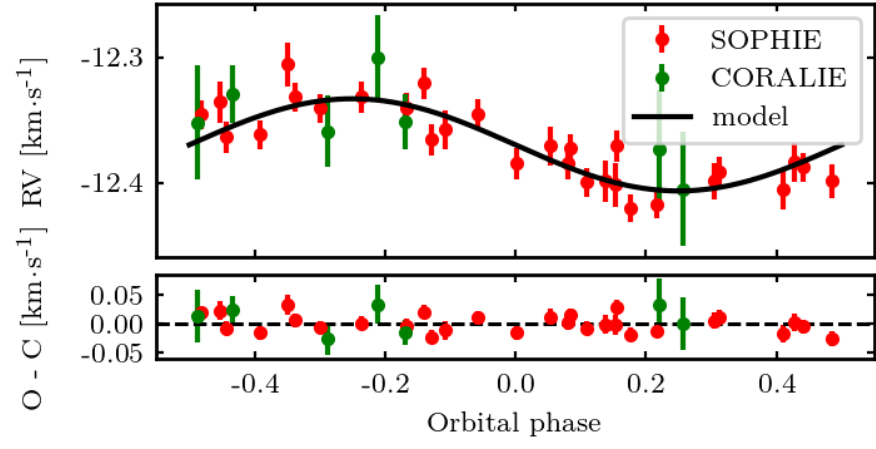

Fig. 3. Radial velocities of WASP-151. The data points are represented with their associated $1 \sigma$ error bars.
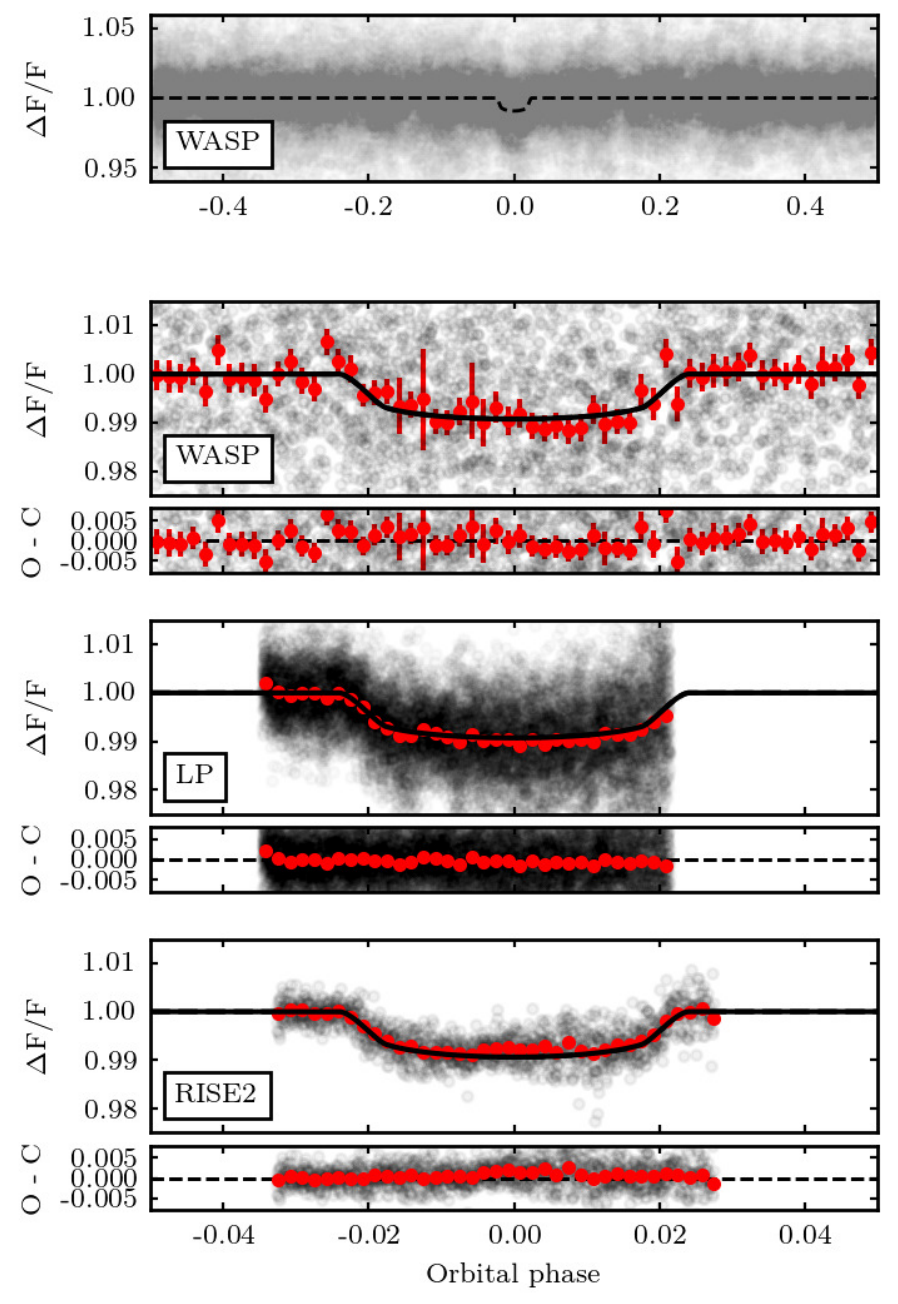

Fig. 4. Photometry of WASP-153. The black or gray points are the data points at the original cadence of the observations, displayed without error bars for clarity. The red points corresponds to the same data points binned in phase with a bin width equivalent to eight minutes. These points are represented with their associated $1 \sigma$ error bars. The black dashed and solid lines correspond to the best-fit model at the original and binned cadence respectively.

instrument to account for a possible bias in the data's error bars due to overestimated, underestimated or even non-considered sources of noise (see Baluev 2009). Finally, we added a parameter for the shift of the radial velocity zero point between two instruments $(\Delta \mathrm{RV})$ and three coefficients to model a linear 


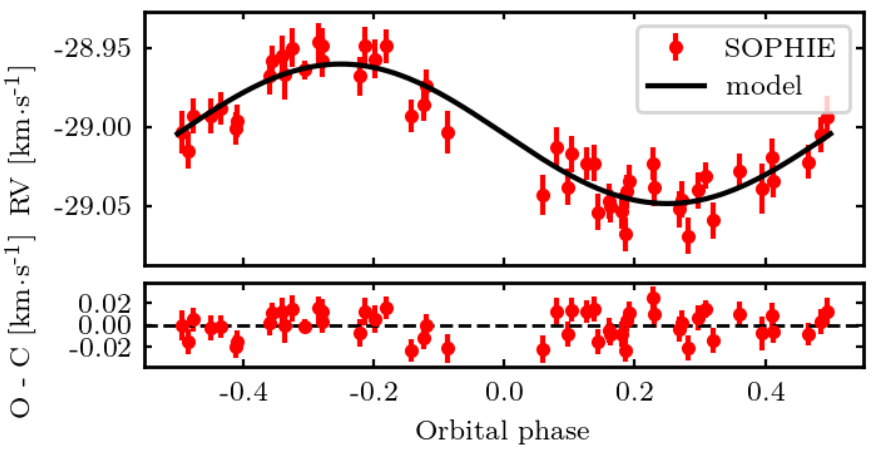

Fig. 5. Radial velocities of WASP-153. The data points are represented with their associated $1 \sigma$ error bars.
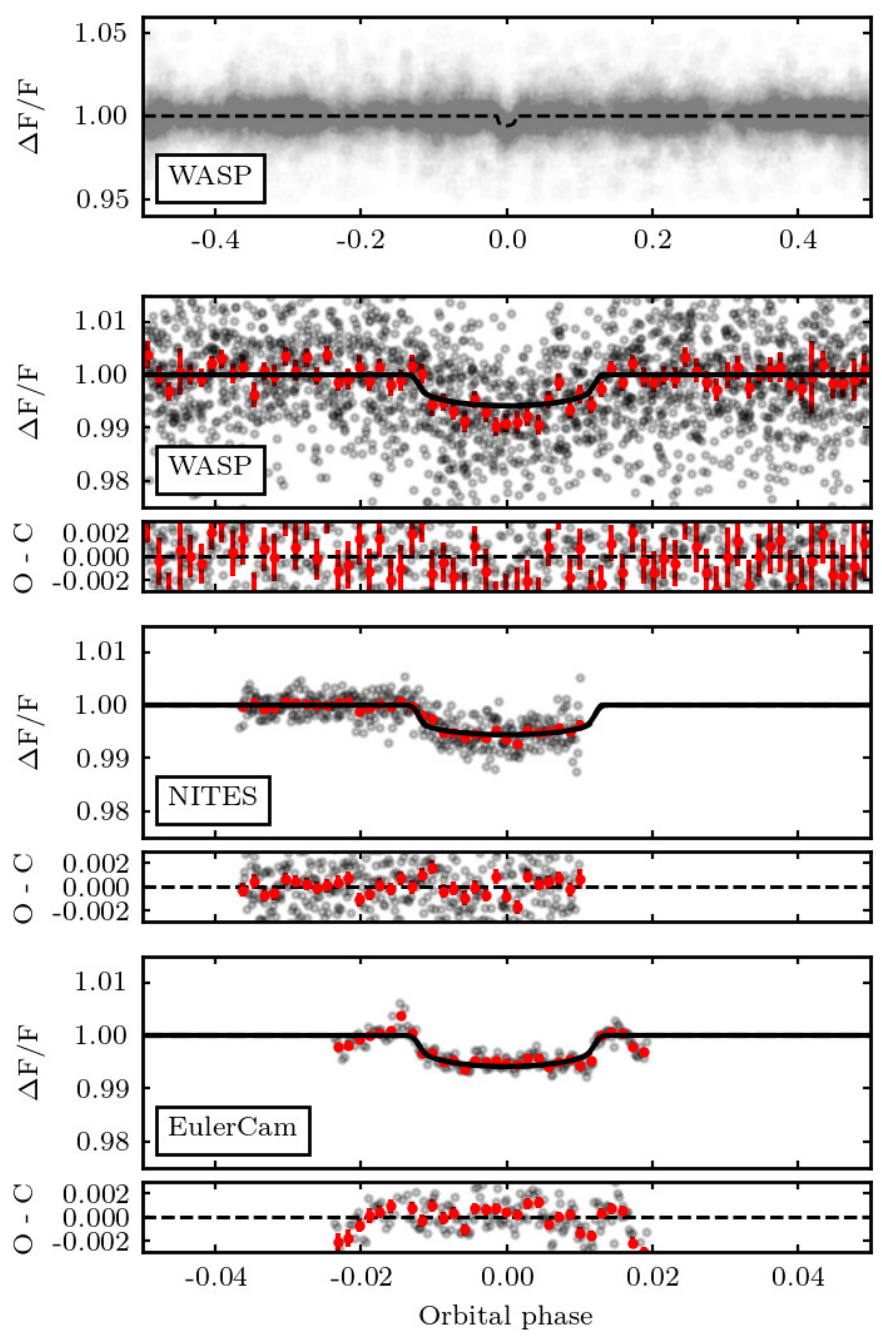

Fig. 6. Photometry of WASP-156. The black or gray points are the data points at the original cadence of the observations, displayed without error bars for clarity. The red points corresponds to the same data points binned in phase with a bin width equivalent to eight minutes. These points are represented with their associated $1 \sigma$ error bars. The black dashed and solid lines correspond to the best-fit model at the original and binned cadence respectively.

or quadratic variation of the out-of-transit relative flux $\left(\Delta F_{\text {OOT }}\right.$, $\Delta F_{\text {OOT }}^{\prime}$ and $\Delta F_{\text {OOT }}^{\prime \prime}$ ) when it was necessary. The final list of main parameters is $R_{\mathrm{p}} / R_{*}, P, t_{c}, \sqrt{e} \cos \omega_{*}, \sqrt{e} \sin \omega_{*}, K, \cos i, a / R_{*}$, $v 0, u$ and $v, \ln f_{\sigma}, \Delta \mathrm{RV}, \Delta F_{\text {OОТ }}, \Delta F_{\text {OОТ }}^{\prime}$ and $\Delta F_{\text {OОT }}^{\prime \prime}$

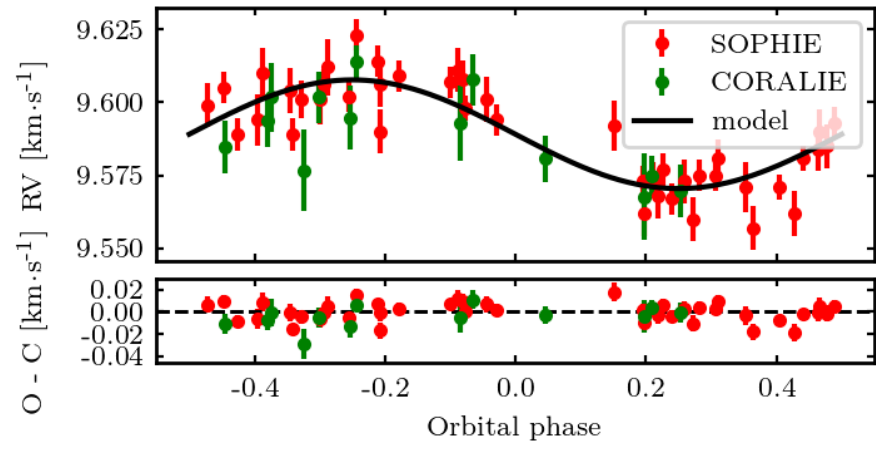

Fig. 7. Radial velocities of WASP-156. The data points are represented with their associated $1 \sigma$ error bars.

To infer accurate values for these parameters, we used the maximum a posteriori (MAP) estimator of the Bayesian inference framework (e.g., Gregory 2005). The prior probability density functions (PDF) assumed for the parameters are given by Table A.5. Along with the posterior PDF provided in Table A.4, it allows for a qualitative assessment of the impact of the prior on the inferred values.

The prior on the limb darkening coefficients deserves a specific consideration. We used Gaussian PDF $s$ whose first two moments were defined using the Python package $1 \mathrm{dtk}{ }^{12}$ (Parviainen \& Aigrain 2015). Using a library of synthetic stellar spectra, it computes the limb darkening profile of a star, observed in a given spectral bandpass (specified by its transmission curve), and defined by its $T_{\text {eff }}, \log g$ and $[\mathrm{Fe} / \mathrm{H}]$. Provided the values and error bars for these stellar parameters and the spectral bandpass, ldtk uses a Markov chain Monte Carlo (MCMC) algorithm to infer the mean and standard deviation of the Gaussian PDF $s$ for the coefficients of a given limb-darkening law (quadratic in our case). ldtk relies on the library of synthetic stellar spectra generated by Husser et al. (2013). It covers a wavelength range, from $500 \AA$ to $5.5 \mu \mathrm{m}$, and a stellar parameter space delimited by: $2300 \mathrm{~K} \leq T_{\text {eff }} \leq 12000 \mathrm{~K}, 0.0 \leq \log g \leq+6.0$, $-4.0 \leq[\mathrm{Fe} / \mathrm{H}] \leq+1.0$, and $-0.2 \leq[\alpha / \mathrm{Fe}] \leq+1.2$. This parameter space is well within the requirements of this study (see Tables 1 and A.4)

The likelihood functions used are multi-dimensional Gaussians, including logarithmic multiplicative jitter factors as described by Baluev (2009). To estimate the MAP and infer reliable error bars, we explored the parameter space using an affineinvariant ensemble sampler for MCMC thanks to the Python package emcee $^{12}$ (see Foreman-Mackey et al. 2013; Hou et al. 2012). We adapted the number of walkers to the number of free parameters in our model. As a compromise between the speed and the efficiency of the exploration, we chose to use $\left\lceil n_{\text {free }} \times\right.$ $2.5 \times 2\rceil / 2$ walkers, where $n_{\text {free }}$ is the number of free parameters and \lceil\rceil the ceiling function. This allows us to have an even number of walkers which is at least twice ( 2.5 times) the number of free parameters, as suggested by Foreman-Mackey et al. (2013).

The introduction in the model of a multiplicative jitter factor complicated the exploration of the parameter space since it introduced local maxima. For the affine-invariant ensemble sampler MCMC algorithm implemented by emcee, when different chains converge toward different disconnected maxima, the exploration becomes less efficient (the acceptance fraction of the chain decreases). Consequently, we separated the exploration into two phases. In a first exploration, we used values randomly generated from the priors as initial values for the free parameters. This first exploration allowed us to locate several (usually two) local 
maxima, to extract the global maximum (the one with the highest posterior probability) and to estimate its location and $68 \%$ confidence level interval. Then we ran a second exploration to precisely sample the global maximum. For this one, the initial values were randomly generated with normal distributions whose mean and standard deviation were set accordingly to the location and width of the global maximum found by the previous step. The final best-fit values for each parameter were estimated from this second exploration after removing any residual burnin phase with the Geweke algorithm (see Geweke et al. 1992). The MAP value for each parameter was finally estimated with the 50th percentile of the associated marginal posteriordistribution. The extrema of the $68 \%$ confidence level intervals were estimated with the 16th and 84th percentiles. These values are reported in Table A.4.

In Table A.4, we also reported the MAP and the $68 \%$ confidence level interval for the secondary parameters. As opposed to the main (or jumping) parameters described in the first paragraph of this section, secondary parameters are not used in the parametrisation chosen for our modeling and are not necessary to perform the MCMC exploration. However, they provide quantities that can be computed from main parameter's values and are of interest to describe the system. The secondary parameters that we computed were: $\Delta F / F$ the transit depth, $i$ the orbital inclination, $e$ the eccentricity, $\omega$ the argument of periastron, $b$ the impact parameter, $D 14$ the outer transit duration (duration between the 1st and 4th contact), D23 the inner transit duration (duration between the 2 nd and 3 rd contact), $R_{\mathrm{p}}$ the planetary radius, $M_{\mathrm{p}}$ the planetary mass, $a$ the semi-major axis, $\tau_{\text {circ }}$ the timescale for the circularisation of the orbit, $F_{i}$ the incident flux on the top of the planetary atmosphere, $T_{\text {eq }}$ the equilibrium temperature of the planet (assuming an albedo of 0 ), $H$ the scale height of the atmosphere assuming a mean molecular weight of $2.2 \mathrm{~g} / \mathrm{mol}, \rho_{*}$ the stellar mean density and $\log g$ the stellar $\log$ gravity. Both $\rho_{*}$ and $\log g$ are, in this case inferred from the transit profile ${ }^{13}$. These estimates are marked with (tr.) in Table A.4. After the full MCMC analysis, we computed the value of all these secondary parameters from the main parameters values and at each step of each walker of the second emcee exploration. Then we estimated their MAP and $68 \%$ confidence level interval with the same method than the main parameters.

The specificities for the analysis of each system were:

WASP-151: the exposure times of the WASP, IAC80, EulerCam and TRAPPIST data are all below $90 \mathrm{~s}$ which is negligible compared to the time scale of the transit variations (typically $30 \mathrm{~min}$ for the transit ingress and egress). However the exposure time of the K2 light curve is $29.424 \mathrm{~min}$. Consequently, for the model of the $\mathrm{K} 2$ data, we supersampled ${ }^{14}$ the model by a factor of ten. This means that for each exposure, we computed the instantaneous value predicted by the model at ten different times evenly distributed over the exposure and then used the average of these ten values as the model value for the whole exposure.

A first analysis of this system showed a linear trend in the residuals of the TRAPPIST and the September IAC80 light curves. We also noticed a more complex behavior in the November IAC80 light curves that we decomposed into two linear trends with a break point at $t=2457328.5022$ HJD. Therefore we split the November IAC80 light curves into two and

\footnotetext{
13 To obtain $\log g$, we also used the estimate of the stellar mass obtained in the next Sect. 3.2.2.

${ }^{14}$ We refer the reader to Kipping (2010) for more details regarding the need of supersampling in light-curve modeling.
}

added 8 parameters to our model to account for these linear variations of the out-of-transit (two per light curve). When doing so, we used the time of the first sample $\left(t_{\min }\right)$ as the origin for the linear function: $\Delta F_{\text {OOT }}+\left(t-t_{\min }\right) \Delta F_{\text {OОT }}^{\prime} \cdot t_{\text {min }}$ is equal to $2457187.753440000124,2457269.443920060061$, $2457328.353823559824,2457328.502696809825$ HJD for the TRAPPIST, the September IAC80, the first part and the second part of the November IAC80 light curves respectively.

We re-analyzed jointly all the datasets with these eight additional free parameters in our model. The inferred parameter values and error bars are reported in Table A.4. Figures 2 and 3 show the photometric and radial velocity data phase folded at the best-fit ephemeris (see Table A.4) with the best-fit model and residuals. The error bars displayed take into account the best-fit jitter values obtained by the Bayesian inference (see Table A.4).

WASP-153: the exposure times of the WASP, Liverpool and RISE-2 data being below $40 \mathrm{~s}$, no supersampling was required for this system. The analysis did not show any abnormal behavior. The inferred parameter values and error bars are reported in Table A.4 and the Figs. 4 and 5 show the photometric and radial velocity data phase folded at the best-fit ephemeris (see Table A.4) with the best-fit model and residuals. The error bars displayed take into account the best-fit jitter values obtained by the Bayesian inference (see Table A.4).

WASP-156: the exposure times of the WASP and NITES data being both below $40 \mathrm{~s}$, no supersampling has been applied for those two datasets. The exposure time of the EulerCam data being around $80 \mathrm{~s}$ and the ingress and egress for this system being relatively short $(\sim 10 \mathrm{~min})$, we decided to supersample the model by a factor of four.

A first analysis of this system showed that the two datasets collected with EulerCam were not compatible. The $2014 \mathrm{Eu}$ lerCam dataset displayed a very pronounced V-shape that was not supported by the other datasets. As described in Sect. 2.2.1, this dataset was identified earlier as being affected by large PSF variations, and stellar counts in the non-linear regime of the $E \mathbf{E}$ lerCam CCD. So we decided to discard it from the final analysis. We also noticed that the residuals of the 2016 EulerCam light curve seemed to exhibit a quadratic trend and introduced three additional parameters to our model to account for a possible quadratic variation of the out-of-transit level. When doing so, we used the time of the first sample $\left(t_{\min }=2457700.517166\right.$ HJD) as the origin for the quadratic function: $\Delta F_{\mathrm{OOT}}+\left(t-t_{\mathrm{min}}\right) \Delta F_{\mathrm{OOT}}^{\prime}+$ $\left.\left(t-t_{\min }\right)^{2} \Delta F_{\text {OOT }}^{\prime \prime \prime}\right)$.

We re-analyzed jointly all the datasets with these three additional free parameters in our model. The inferred parameter values and error bars are reported in Table A.4. Figures 6 and 7 show the photometric and radial velocity data phase folded at the best-fit ephemeris (see Table A.4) with the best-fit model and residuals. The error bars displayed take into account the best-fit jitter values obtained by the Bayesian inference (see Table A.4).

\subsubsection{Stellar modeling}

In Sect. 3.1, we derived stellar masses and radii from $T_{\text {eff }}$, $\log g$ and $[\mathrm{Fe} / \mathrm{H}]$ using the Torres et al. (2010) calibration and ages using lithium abundances and gyrochronology. If those two age estimates seem to agree for our three systems, the lithium constraint on the age is very weak and gyrochronology is known to sometimes contradict other age estimators such 
as isochronal ages (e.g., Buzasi et al. 2016; Angus et al. 2015; Kovács 2015; Maxted et al. 2015b). Furthermore, the additional constraint brought by the stellar density inferred from the transit and a dedicated modeling of the star should result in more accurate estimates of the stellar masses and radii. Consequently to provide a more comprehensive view of our three systems, we modeled the stars using the Fortran software bagemass ${ }^{15}$ (Maxted et al. 2015a).

Bagemass relies on a grid of stellar models ${ }^{16}$ produced with the GARSTEC stellar evolution code (Weiss \& Schlattl 2008). This grid covers the mass range between 0.6 to $2.0 M_{\odot}$, the initial metallicity range between -0.75 to $0.55 \mathrm{dex}$ and the age range between the end of the pre-main-sequence phase up to $17.5 \mathrm{Gyr}$ (or a maximum radius of $3 R_{\odot}$ depending on which one occurs first). In order to obtain stellar properties for any mass, metallicity and age within these ranges, and not only for the points in the grid, bagemass uses the cubic spline interpolation algorithm PSPLINE ${ }^{17}$. Given measurements (values and error bars) for the $T_{\text {eff }},[\mathrm{Fe} / \mathrm{H}]$ and density $\left(\rho_{*}\right)$ of the star studied, it then explores this parameter space using a MCMC method which computes the posterior probability as a function of mass and age.

Using the $T_{\text {eff }}$ and $[\mathrm{Fe} / \mathrm{H}]$ estimates provided by the spectral analysis and the stellar density estimates obtained from the analysis of the transit (see Sect. 3.2 and Table A.4), we obtained estimates and $68 \%$ confidence interval error bars for the ischronal age and the mass of our three stars ${ }^{18}$. These values are reported in Table A.4. Figure 8 shows the marginalized probability distribution in the Hertzsprung-Russell diagram along with the best-fit evolutionary model and isochrones for our three stars. To provide more robust error bars, the error provided in Table A.4 for the mass estimate $\left(M_{*}(t r .+e v\right.$. track) $)$ is the square-root of the quadratic sum of the internal error and the sensitivities to the mixing length parameter and the helium-enhancement. Finally, we also computed new estimates for the secondary parameters of the transit and RV analysis (see Sect. 3.2.1) which rely on the stellar mass and radius estimates. The most sensitive of those parameters are $R_{p}, M_{p}, \rho_{p}, H$ and $F_{i}$. We reported these estimates in Table A.4.

The interpretation of the isochronal age estimate is the subject of Sect. 4.3, so we will now focus on the stellar mass and radius estimates. For WASP-151, this analysis provides estimates that are compatible within one sigma with the ones obtained with the Torres et al. (2010) calibration (Sect. 3.1). However for WASP-153 and WASP-156, it's not the case. The stellar modeling predicts a significantly bigger radius for WASP-153 and a significantly lower radius for WASP-156 while the masses are compatible within one sigma (see Table A.4). This difference is mainly explained by the difference in $\log g$ between the spectroscopic and transit analyses (see tr. and spec. values of $\log g$ in Table A.4). The comparison of $\log g$ estimates from spectroscopy made by Smalley (2005) showed that a realistic error bars for a $\log g$ estimator from spectroscopy is $\sim 20 \%$, while the one inferred from the transit density is more direct and more

\footnotetext{
${ }^{15}$ We used the version 1.1 available at http://sourceforge.net/ projects/bagemass

${ }^{16}$ bagemass provides several grids with different mixing length $\left(\alpha_{\mathrm{MLT}}\right.$ equals 1.78 or 1.50 ) and different helium-enhancement (0.0 or 0.2$)$. For this work, we used the default values which correspond to no heliumenhancement and $\alpha_{\mathrm{MLT}}=1.78$. However, in Table A.6, we present estimates of the sensitivity of the results to this assumptions.

17 The PSPLINE algorithm is available at http://w3.pppl.gov/ ntcC/PSPLINE

${ }^{18}$ The complete output table provided by bagemass is available in Table A.6.
}

robust with typical uncertainties $\$ 5 \%$ depending on the quality of the light curve and the photometric stellar variability. As described in Sect. 2.1, our three stars are not particularly active. We will thus rely on the stellar mass and radius estimates obtained in this section for the rest of the paper, even if we show in Figs. 9, 10, and Table A.4 the estimates which rely on the spectroscopic $\log g$ for completeness.

\section{Discussion and conclusion}

Table A.4 gives us an exhaustive picture of these three systems and allows us to put them in context. WASP-151b and WASP$153 \mathrm{~b}$ are relatively similar. Their masses of 0.31 and $0.39 M_{\text {Jup }}$ and semi-major axes of $0.056 \mathrm{AU}$ and $0.048 \mathrm{AU}$ respectively indicate two Saturn-size objects around early $G$ type stars of $V$ magnitude $\sim 12.8$. WASP-156b's radius of $0.51 R_{\text {Jup }}$ suggests a super-Neptune $^{19}$ and makes it the smallest planet ever detected by WASP. Its mass of $0.128 M_{\text {Jup }}$ is also the third lightest planet detected by WASP after WASP-139b (Hellier et al. 2017) and WASP-107b (Anderson et al. 2017). It is also interesting that WASP-156 is a bright ( $\operatorname{mag} V=11.6) \mathrm{K}$ type star.

In the following two sections, we compared the position of our three planets in the mass-radius diagram with the isochrones of Baraffe et al. (2008) to constrain their composition. Baraffe et al. (2008) provide two types of models, one without irradiation and one with the irradiation received at $0.045 \mathrm{AU}$ from the Sun. Given the semi-major axes of our planets, the latter is the most suited to this study and is the one we used in Fig. 9. We refer readers interested in the details of these models to the associated publication.

In the third section, we discuss the age estimates of those three systems. More specifically, we address the apparent discrepancy between the gyrochronological and isochronal ages and the possible insight that it provides regarding the migration mechanism of the planets in these systems. Finally, the fourth section is devoted to the impact of these three planets on our understanding of the Neptunian desert (Mazeh et al. 2016).

\subsection{Two hot Saturns: WASP-151b and WASP-153b}

WASP-151b and WASP-153b's positions in the mass-radius diagram indicate two low density gaseous planets (see Fig. 9). Their masses are close to that of Saturn but their radii are significantly bigger, especially for WASP-153b. According to its isochronal age and its relative position compared to the isochrones of Baraffe et al. (2008), WASP-151b should have a heavy-element mass fraction slightly smaller than $2 \%$. Similarly, WASP-153b's heavy-element mass fraction should be significantly smaller than $2 \%$. Knowing that WASP-151 has a metallicity compatible with that of the Sun, that WASP-153 is super-metallic $([\mathrm{Fe} / \mathrm{H}]=$ $0.34 \pm 0.11 \mathrm{dex})$ and that the Sun's heavy element mass fraction is close to $2 \%$ (e.g., Baraffe et al. 2008), these heavy element mass fractions inferior to $2 \%$ are unlikely. Consequently, WASP$151 \mathrm{~b}$ appears to be slightly more bloated than the models predict and WASP-153b exhibits a significant radius anomaly. This interpretation is, of course, dependant on the accuracy of our mass, radius and age estimates. As shown in Fig. 9, if we rely on the planetary radius inferred from the purely spectroscopic stellar parameters (Sect. 3.1), WASP-151b and WASP-153b are compatible within one sigma with the model of Baraffe et al. (2008).

\footnotetext{
19 Bakos et al. (2015) defined the class of super-Neptunes as the planets whose mass lies between $0.054 M_{\text {Jup }}$ (the mass of Neptune) and $0.18 M_{\text {Jup }}$ (halfway between the mass of Neptune and Saturn).
} 

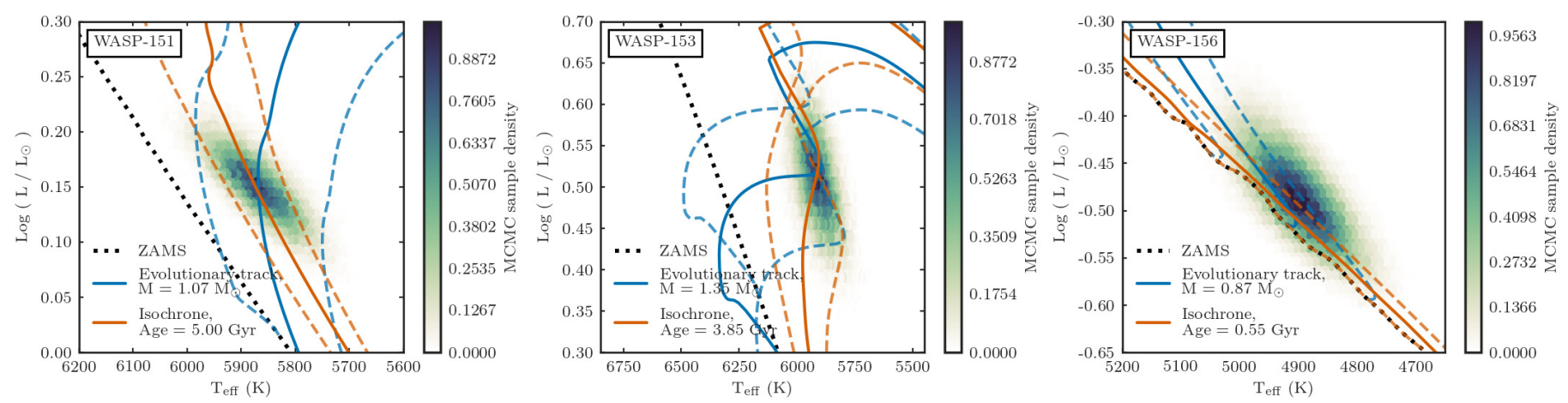

Fig. 8. WASP-151, WASP-153 and WASP-156 marginalized posterior distribution in the Hertzsprung-Russell diagram. The dotted black lines correspond to the zero-age main sequence (ZAMS) at best-fit $[\mathrm{Fe} / \mathrm{H}]$. This is defined as the time at which the star reaches its minimum luminosity and stellar ages are measured relative to this time. The blue lines are stellar mass evolutionary tracks and the orange ones are age isochrones. For both isochrones and evolutionary tracks the solid line correspond to the best-fit model (maximum of joint likelihood distribution) and the dashed lines correspond to the two extrema of the $68 \%$ confidence interval. For more details see Sect. 3.2.2 and Table A.6.

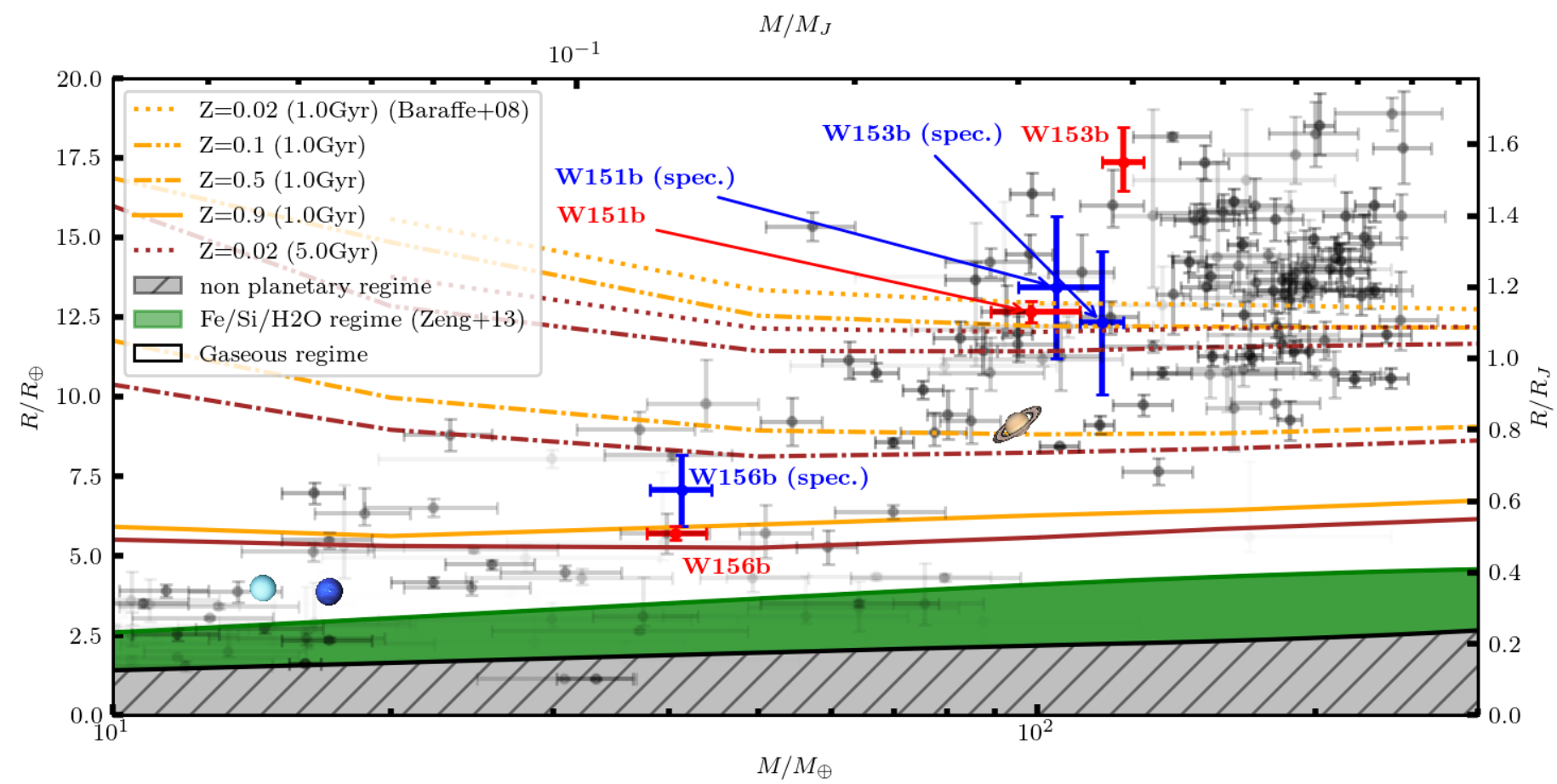

Fig. 9. WASP-151b, WASP-153b and WASP-156b in the mass-radius diagram. The black points with $1 \sigma$ error bars are the known confirmed planets according to exoplanet.eu (Schneider et al. 2011). Their transparency reflect the relative precision on their mass and radius. The better the parameter of a planet are constrained the more opaque the point is. The red and blue points with $1 \sigma$ errors bars are the planets announced by this paper. For the red points the mass and radius estimates rely on stellar parameters obtained via evolutionary tracks and the stellar density inferred from the transit (see Sect. 3.2.2), while for the blue points, they rely on purely spectroscopic stellar parameters (see Sect. 3.1). The solar system planets (Saturn, Uranus and Neptune) have also been reported in this diagram for reference. The two shaded areas at the bottom of the graph define the non-planetary regime (gray striped) and the rocky-water world regime (green) as defined by Zeng \& Sasselov (2013). Consequently the rest of the diagram represents the gaseous-ice giant regime. The solid, dotted and dashed lines represent the mass-radius relations for gaseous planets of different age and different heavy element mass fraction $(Z)$ as described by (Baraffe et al. 2008). The type of line (solid, dashed, or dotted) represents the heavy element mass fraction and the color of the line represents the age. These models have been used to constrain the nature and composition of WASP-151b, WASP-153b and WASP-156b, see Sects. 4.1 and 4.2.

However as discussed in Sect. 3.2.2, these estimates appear less precise and less accurate than the ones above, which rely on the stellar densities inferred from the transit and stellar models.

Given the relatively high incident flux received by these two planets (460 $F_{\mathrm{i}, \oplus}$ for WASP-151b and $1400 F_{\mathrm{i}, \oplus}$ for WASP$153 \mathrm{~b}$ ), the radius anomalies that they exhibit was expected. Indeed, it is in agreement with the empirical thresholds defined by Miller \& Fortney (2011) and Lopez \& Fortney (2016) for an abnormally inflated radius: $R>1.2 R_{\mathrm{Jup}}$ and $F_{\mathrm{i}}>$ $2 \times 10^{8} \mathrm{erg} \mathrm{s}^{-1} \mathrm{~cm}^{-2} \sim 150 F_{\mathrm{i}, \oplus}$. WASP-153b significantly exceeds both thresholds and WASP-151b exceeds the incident flux threshold, but is slightly below the radius threshold.

\subsection{A warm super-Neptune: WASP-156b}

WASP-156b's position in the mass-radius diagram suggests a composition significantly different from the ones of WASP-151b and WASP-153b. Baraffe et al. (2008) models indicate a high heavy element mass fraction around $90 \%$, in agreement with the one of Neptune and Uranus (Helled \& Guillot 2017), depicting 
WASP-156b as a warm super-Neptune. Super-Neptunes with precise determination of the mass and radius (better than $15 \%$ ) are relatively rare since only nine of these objects are known at the moment: Kepler-9c (Torres et al. 2011), Kepler-35b (Welsh et al. 2012), Kepler-101b (Bonomo et al. 2014), HATS$7 b$ (Bakos et al. 2015), HATS-8b (Bayliss et al. 2015), WASP107b (Anderson et al. 2017), WASP-127b (Lam et al. 2017), WASP-139b (Hellier et al. 2017), and WASP-156b. Amongst this class of planets, WASP-156b, as a warm $\left(T_{\mathrm{eq}}=970 \mathrm{~K}\right)$ and dense $\left(\rho_{\mathrm{p}}=1.0 \rho_{\text {Jup }}\right)$ super-Neptune, is particularly interesting to investigate the gaseous to ice giant transition as described by Anderson et al. (2017) and Bakos et al. (2015). WASP-156 is also currently the brightest super-Neptune host star, with a $V$ magnitude of 11.6, making it a target of prime interest for future atmospheric characterization.

\subsection{Discrepancy between the ages estimators, an insight on migration mechanisms?}

In Sects. 3.1 and 3.2.2, we derived ages for our three stars with Lithium abundance, gyrochronology and isochrone fitting. These results are reported in Table 3. The tendency that arises from this table is that our stars tend to have isochronological ages that are significantly higher than their gyrochronological ages. This tendency, limited here to three cases, has already been observed by Maxted et al. (2015b) for a broader sample of 28 transiting exoplanets where at least half of the sample exhibits this discrepancy. Interestingly for more than $80 \%$ of the stars in this sample, and for our three stars, the planetary companion is a short-period (less than five days) giant planet.

Discrepancies between gyrochronological and isochronological ages have been reported by several studies and not only in the context of planet host stars, see for example Angus et al. (2015), Kovács (2015), Buzasi et al. (2016). Maxted et al. (2015b) found that gyrochronological age estimates were significantly lower than the isochronological ones for about half of their sample of planetary hosts. Kovács (2015) reached a similar conclusion from a galactic field stars sample. Finally Buzasi et al. (2016) and Angus et al. (2015) brought to light inconsistencies in the gyrochronological age estimator when applied to different samples. This problem is thus complex and has multiple facets. Consequently, it will not be solved solely by the three stars discussed in this paper. However they can give us insights regarding the specific question of the underestimation provided by the gyrochronological age estimator observed for a fraction of the short period planet host stars population.

To explain the hot giant planet population, the core-accretion scenario requires a mechanism to migrate these planets from their formation location, beyond the ice line, to the vicinity of their parent star. There are currently two mechanisms debated in the literature for this migration: disk driven migration (e.g., Lin et al. 1996; Ward 1997) and high eccentricity migration (e.g., Rasio \& Ford 1996; Fabrycky \& Tremaine 2007). The main observational arguments to favor one over the other are: spin-orbit misalignment (e.g., Naoz et al. 2012), stellar metallicity (e.g., Dawson \& Murray-Clay 2013), the presence of additional companions (e.g., Schlaufman \& Winn 2016) and the Roche separation (e.g., Nelson et al. 2017).

In the light of Table 3 and the study performed by Maxted et al. (2015b), we suggest that a gyrochronological age significantly smaller than the isochronal one could be evidence to identify the mechanism responsible for the migration of giant planets. A gyrochronological ages significantly lower than the isochronological one might indeed be explained by the important
Table 3. Age estimates of WASP-151, WASP-153 and WASP-156.

\begin{tabular}{llll}
\hline \hline Star & Iso. [Gyr] & Gyro. [Gyr] & Li \\
\hline WASP-151 & $5.13_{-1.33}^{+1.33}$ & $1.80_{-1.00}^{+2.03}$ & several Gyr \\
WASP-153 & $4.00_{-0.77}^{+0.77}$ & $1.21_{-0.60}^{+1.19}$ & several Myr \\
WASP-156 & $6.50_{-4.03}^{+4.03}$ & $0.58_{-0.31}^{+0.51}$ & $\gtrsim 500 \mathrm{Myr}$ \\
\hline
\end{tabular}

Notes. Iso. stands for isochronal age, Gyro. for gyrochronological age and $\mathrm{Li}$ for the age constraint based and lithium abundance. The isochronal age estimates in this table are obtained using the mean value of the marginalized posterior distribution of the age. For WASP-151 and WASP-153, these are compatible with the maximum-likelihood estimate. However for WASP-156, it is not the case since the latter give an age of $0.5 \mathrm{Gyr}$ (see Table A.6).

transfer of angular momentum from the giant planet to the star during the tidal circularisation of the planet's orbit involved in high eccentricity migration. On the contrary, disk driven migration implies an exchange of angular momentum between the planet and the disk and cannot directly explain an increase of the stellar rotation. Furthermore, contrary to disk driven migration, high eccentricity migration is not bounded to the short protoplanetary disk lifetime and can occur at an older stage of the system amplifying even more the discrepancy between the two age estimates. If this hypothesis is confirmed for stars hosting short period planets, a gyrochronological age significantly smaller the isochronal age (e.g., the three host star presented in this paper) would indicate that the planet migrated through higheccentricity migration while a gyrochronological age compatible with the isochronal one (e.g., WASP-33; Collier Cameron et al. 2010) would suggest a disk driven migration (or an in-situ formation).

Obviously, a more thorough analysis is necessary to investigate all the possible implications behind this hypothesis. Such an analysis is beyond the scope of this paper but we think that this hypothesis is worth investigating. In this context, a search for long period companions that might have triggered the high eccentricity migration or an independent age estimate through asterosismology with TESS (Campante et al. 2016) or Plato (Rauer et al. 2014) would be particularly interesting.

\subsection{Three planets at the border of the Neptunian desert}

As described in the introduction, Mazeh et al. (2016) studied the distribution of the planet population in the orbital period, mass and radius domain and reported the lower and upper mass and radius boundaries of the short period Neptunian desert. Figure 10 shows that WASP-151b and WASP-153b lie near the upper boundaries of the desert, while WASP-156b stands well inside it. The authors mentioned that the period limit of the desert was not well constrained, however they also indicated that these borders delineate the boundaries for periods below five days, which is the case of WASP-156b. Understanding the differences between WASP-156b on the one side and WASP-151b and WASP-153b on the other side might allow us to shed light on the mechanism responsible for the upper boundary of the Neptunian desert. Mazeh et al. (2016) proposed two explanations for the origin of the upper boundary of the desert:

- Gaseous planets cannot exist below the upper boundary, because they would lose their gaseous envelope due to stellar insolation (e.g., Lopez \& Fortney 2014) or Roche-lobe overflow (e.g., Kurokawa \& Nakamoto 2014). 

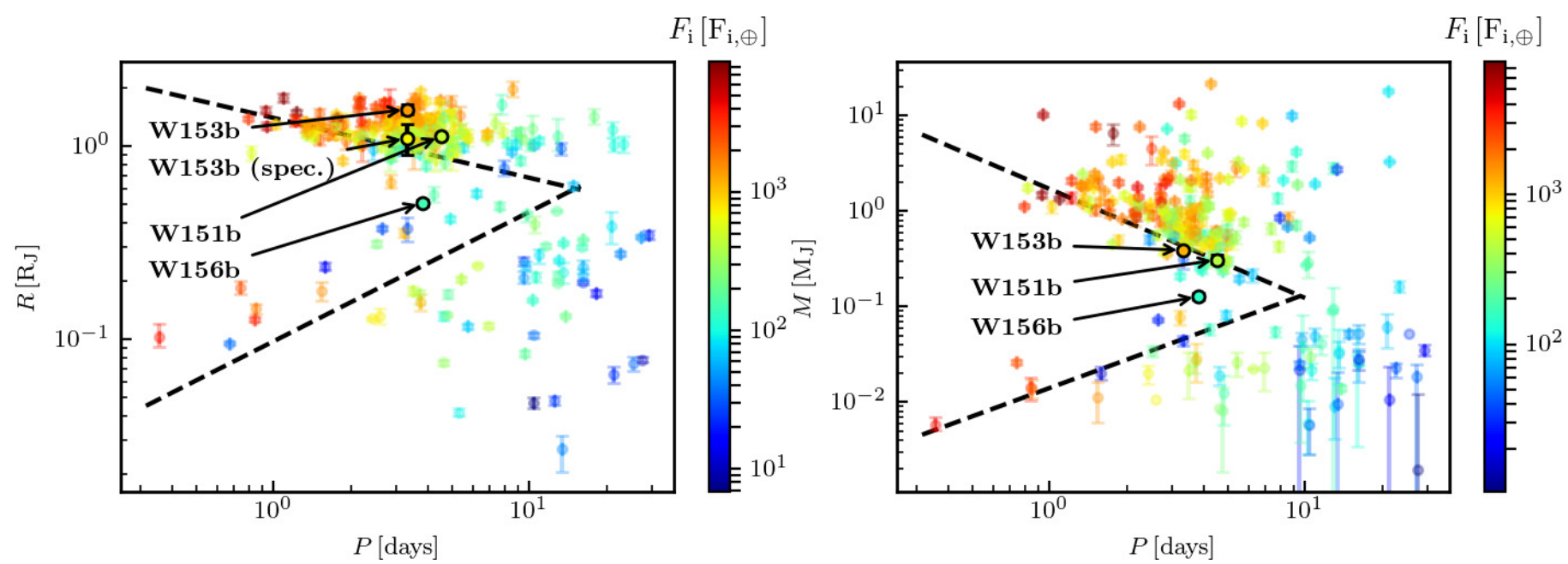

Fig. 10. WASP-151b, WASP-153b and WASP-156b in the radius versus orbital period (left) and the mass versus orbital period (right) domains. The colored points correspond to the known exoplanet and the color reflect their bolometric incident flux. WASP-151b, WASP-153b and WASP-156b are circled in black and the black dashed lines correspond to the upper and lower boundaries of the Neptunian desert as reported by Mazeh et al. (2016), see Sect. 4.4 for details.

- Gaseous planets are formed further away from their parent star and cannot migrate below the upper boundary, because at this distance from the star the disk is not dense enough to sustain inward migration.

While a detailed analysis of the origin of the Neptunian desert is beyond the scope of this paper, it is still interesting to look into the similarities and differences between WASP-156b and WASP-151b/WASP-153b since they might provide useful insights on the nature of this desert. These three planets possess similar orbital parameters (see Table A.4). Their ages are subject to caution (as discussed in Sect. 4.3), but a given estimator provides similar ages for these three stars. Their gyrochronological ages indicate relatively young systems $(\sim 1 \mathrm{Gyr}$ for WASP-151 and WASP-153 and $\sim 0.5$ Gyr for WASP-156), while their isochronal ages indicate $\sim 5$ Gyr old systems. However, their radiative environments are significantly different. WASP-151b and WASP-153b receive a higher bolometric irradiation $\left(460,1400\right.$ and $150 F_{\mathrm{i}, \oplus}$ for WASP-151b, WASP153b and WASP-156b respectively). Moreover, the spectral types of their host stars are different (early G for WASP-151 and WASP-153 and early $\mathrm{K}$ for WASP-156) implying a different spectral content of the irradiation, especially in extreme ultra-violet (EUV). The EUV flux is particularly interesting in this context since it is the main contributor for exoplanet atmosphere evaporation. Lecavelier Des Etangs (e.g., 2007) provided estimates for the EUV flux emitted by stars of different spectral types. According to these estimates, the EUV flux received by WASP$156 \mathrm{~b}$ is approximately three times higher than the one received by WASP-151b and WASP-153b, $F_{\text {EUV@ } 1 \mathrm{AU}}$ is $15 \mathrm{erg} \mathrm{cm}^{-2} \mathrm{~s}^{-1}$ for $\mathrm{K}$ type stars and $5 \mathrm{erg} \mathrm{cm}^{-2} \mathrm{~s}^{-1}$ for $\mathrm{G}$ type stars. This suggests that photo-evaporation is the mechanism responsible for the presence of WASP-156b below the upper boundary of the short-period Neptunian desert. WASP-156 may be in the process of losing its gaseous envelope in a short-lived evolutionary phase which places it within the underpopulated short-period Neptunian desert.

Finally, in the context of the hypothesis formulated in Sect. 4.3 , it is also interesting to mention the alternative explanation defended by Matsakos \& Königl (2016) for the origin of the Neptunian desert. The authors present the desert as the result of high-eccentricity migration of planets that arrive in the vicinity of the Roche limit of their host star and suggest that the slopes and positions of the upper and lower boundaries are a direct consequence of the different mass-radius relations for rocky and gaseous planets.

Acknowledgements. O.D.S.D acknowledges the support from Fundação para a Ciência e a Tecnologia (FCT) through national funds and by FEDER through COMPETE2020 by grants UID/FIS/04434/2013\&POCI-01-0145-FEDER007672 and PTDC/FIS-AST/1526/2014\&POCI-01-0145-FEDER-016886. S.C.C.B. also acknowledges support from FCT through Investigador FCT contract IF/01312/2014/CP1215/CT0004. C.A.H. was supported by STFC under grant ST/P000584/1. A.S.B. acknowledges funding from the European Union Seventh Framework programme (FP7/2007-2013) under grant agreement No. 313014 (ETAEARTH). D.J.A.B acknowledges funding from the UKSA and the University of Warwick. F.F. acknowledges financial support from "Accordo ASI-INAF for PLATO" No. 2015-019-R.0 July 29, 2015. L.D. acknowledges support from the Gruber Foundation Fellowship. The SuperWASP Consortium consists of astronomers primarily from University of Warwick, Queens University Belfast, St. Andrews, Keele, Leicester, The Open University, Isaac Newton Group La Palma and Instituto de Astrofísica de Canarias. The SuperWASP-N camera is hosted by the Issac Newton Group on La Palma and WASPSouth is hosted by SAAO. We are grateful for their support and assistance. Funding for WASP comes from consortium universities and from the UK's Science and Technology Facilities Council. The research leading to these results has received funding from the European Community's Seventh Framework programmes (FP7/2007-2013 and FP7/2013-2016) under grant agreement number RG226604 and 312430 (OPTICON), respectively. This work is based on radial velocity observations made at Observatoire de Haute Provence (CNRS), France and at ESO in La Silla (Chile) with the CORALIE Echelle spectrograph mounted on the Swiss telescope. We thank the staff at Haute-Provence Observatory. TRAPPIST is funded by the Belgian Fund for Scientific Research (Fond National de la Recherche Scientifique, F.R.S.-FNRS) under the grant FRFC 2.5.594.09.F, with the participation of the Swiss National Science Fundation (SNF). The research leading to these results has received funding from the ARC grant for Concerted Research Actions, financed by the Wallonia-Brussels Federation. M.G. and E.J. are, respectively, Research Associate and Senior Research Associate at the F.R.S.-FNRS. The Swiss Euler Telescope is a project financed by the Swiss National Science Foundation. The Liverpool Telescope is operated on the island of La Palma by Liverpool John Moores University in the Spanish Observatorio del Roque de los Muchachos of the Instituto de Astrofisica de Canarias with financial support from the UK Science and Technology Facilities Council. The Aristarchos telescope is operated on Helmos Observatory by the Institute for Astronomy, Astrophysics, Space Applications and Remote Sensing of the National Observatory of Athens. This paper includes data collected by the $\mathrm{K} 2$ mission. Funding for the $\mathrm{K} 2$ mission is provided by the NASA Science Mission directorate. Used Simbad, Vizier, exoplanet.eu. The authors also want to thank Pedro Figueira, Nuno Santos and Mahmoud Oshagh for fruitful discussions and Joao Faria for his Python figure styler. Most of the analyses presented in this paper were performed using the Python language (version 3.5) available at http://wwW.python.org and several scientific 
packages: Numpy \& Scipy (Van Der Walt et al. 2011), Pandas (McKinney et al 2010), Ipython (Perez et al. 2007), Astropy (Astropy Collaboration et al. 2013) and Matplotlib (Hunter 2007).

\section{References}

Angus, R., Aigrain, S., Foreman-Mackey, D., \& McQuillan, A. 2015, MNRAS, 450,1787

Anderson, D. R., Collier Cameron, A., Delrez, L., et al. 2017, A\&A, 604, A110 Asplund, M., Grevesse, N., Sauval, A. J., \& Scott, P. 2009, ARA\&A, 47, 481 Astropy Collaboration, Robitaille, T. P., Tollerud, E. J., et al. 2013, A\&A, 558, A33

Baluev, R. V. 2009, MNRAS, 393, 969

Bakos, G. Á., Penev, K., Bayliss, D., et al. 2015, ApJ, 813, 111

Baraffe, I., Chabrier, G., \& Barman, T. 2008, A\&A, 482, 315

Baranne, A., Queloz, D., Mayor, M., et al. 1996, A\&AS, 119, 373

Barnes, S. A. 2007, ApJ, 669, 1167

Barentsen, G. 2017, DOI: 10.5281/zenodo.344973

Barros, S. C. C., Faedi, F., Collier Cameron, A., et al. 2011, A\&A, 525, A54

Barros, S. C. C., Demangeon, O., \& Deleuil, M. 2016, A\&A, 594, A10

Bayliss, D., Hartman, J. D., Bakos, G. Á., et al. 2015, AJ, 150, 49

Benz, W., Ehrenreich, D., Broeg, C., et al. 2013,

http://sci.esa.int/cosmic-vision/53541-cheops-definitionstudy-report-red-book/\#

Boisse, I., Eggenberger, A., Santos, N. C., et al. 2010, A\&A, 523, A88

Bonomo, A. S., Sozzetti, A., Lovis, C., et al. 2014, A\&A, 572, A2

Borucki, W. J. 2017, Proc. Am. Phil. Soc., 161, 38

Borucki, W. J., Koch, D., Basri, G., et al. 2010, Science, 327, 977

Bouchy, F., Hébrard, G., Udry, S., et al. 2009, A\&A, 505, 853

Boumis, P., Pollacco, D., Steele, I., et al. 2010, 9th International Conference of the Hellenic Astronomical Society, 424, 426

Buzasi, D., Lezcano, A., \& Preston, H. L. 2016, J. Space Weather Space Clim., 6, A38

Campante, T. L., Schofield, M., Kuszlewicz, J. S., et al. 2016, ApJ, 830, 138

Collier Cameron, A., Pollacco, D., Street, R. A., et al. 2006, MNRAS, 373, 799

Collier Cameron, A., Wilson, D. M., West, R. G., et al. 2007, MNRAS, 380 1230

Collier Cameron, A., Guenther, E., Smalley, B., et al. 2010, MNRAS, 407, 507

David, T. J., Hillenbrand, L. A., Petigura, E. A., et al. 2016, Nature, 534, 658

Dawson, R. I., \& Murray-Clay, R. A. 2013, ApJ, 767, L24

Díaz, R. F., Almenara, J. M., Santerne, A., et al. 2014, MNRAS, 441, 983

Doyle, A. P., Smalley, B., Maxted, P. F. L., et al. 2013, MNRAS, 428, 3164

Doyle, A. P., Davies, G. R., Smalley, B., Chaplin, W. J., \& Elsworth, Y. 2014 MNRAS, 444, 3592

Eastman, J., Gaudi, B. S., \& Agol, E. 2013, PASP, 125, 83

Espinoza, N., Brahm, R., Jordán, A., et al. 2010, ApJ, 830, 43

Fabrycky, D., \& Tremaine, S. 2007, ApJ, 669, 1298

Fabrycky, D. C., Lissauer, J. J., Ragozzine, D., et al. 2014, ApJ, 790, 146

Faedi, F., Barros, S. C. C., Anderson, D. R., et al. 2011, A\&A, 531, A40

Figueira, P., Faria, J. P., Adibekyan, V. Z., Oshagh, M., \& Santos, N. C. 2016 Origins of Life and Evolution of the Biosphere, 46, 385

Foreman-Mackey, D., Hogg, D. W., Lang, D., \& Goodman, J. 2013, PASP, 125, 306

Geweke J., Bernado J.-M., et al. 1992, Evaluating the accuracy of samplingbased approaches to calculating posterior moments, Bayesian Statistics 4 (Oxford UK: Clarendon Press)

Gillon, M., Jehin, E., Magain, P., et al. 2011, in Detection and Dynamics of Transiting Exoplanets, eds. F. Bouchy, R. Díaz, \& C. Moutou, EPJ Web Conf., 11,6002

Grasset, O., Schneider, J., \& Sotin, C. 2009, ApJ, 693, 722

Gray D. F., 2008, The observation and analysis of stellar photospheres, 3rd edn (Cambridge University Press), 507

Gregory, P. C. 2005, Bayesian Logical Data Analysis for the Physical Sciences (Cambridge University Press)

Hébrard, G., Bouchy, F., Pont, F., et al. 2008, A\&A, 481, 52

Helled, R., \& Guillot, T. 2017, ArXiv e-prints [arXiv: 1705.0932]

Hellier, C., Anderson, D. R., Cameron, A. C., et al. 2017, MNRAS, 465, 3693

Hou, F., Goodman, J., Hogg, D. W., Weare, J., \& Schwab, C. 2012, ApJ, 745, 198

Howell, S. B., Sobeck, C., Haas, M., et al. 2014, PASP, 126, 398

Hunter, J. D. 2007, Comput. Sci. Eng., 9, 90
Husser, T.-O., Wende-von Berg, S., Dreizler, S., et al. 2013, A\&A, 553, A6 Jehin, E., et al. 2011, The Messenger, 145, 2

Kipping, D. M. 2010, MNRAS, 408, 1758

Kovács, G. 2015, A\&A, 581, A2

Kovács, G., Zucker, S., \& Mazeh, T. 2002, A\&A, 391, 369

Kovács, G., Bakos, G., \& Noyes, R. W. 2005, MNRAS, 356, 557

Kreidberg, L. 2015, PASP, 127, 1161

Kurokawa, H., \& Nakamoto, T. 2014, ApJ, 783, 54

Lam, K. W. F., Faedi, F., Brown, D. J. A., et al. 2017, A\&A, 599, A3

Lecavelier Des Etangs, A. 2007, A\&A, 461, 1185

Lendl, M., Anderson, D. R., Collier Cameron, A., et al., 2012, A\&A, 544, A72

Lin, D. N. C., Bodenheimer, P., \& Richardson, D. C. 1996, Nature, 380, 606

Lopez, E. D., \& Fortney, J. J. 2014, ApJ, 792, 1

Lopez, E. D., \& Fortney, J. J. 2016, ApJ, 818, 4

Luger, R., Agol, E., Kruse, E., et al. 2016, AJ, 152, 100

Luger, R., Sestovic, M., Kruse, E., et al. 2017, Nat. Astron., 1, 0129

Lundkvist, M. S., Kjeldsen, H., Albrecht, S., et al. 2016, Nat. Comm., 7, 11201

Matsakos, T., \& Königl, A. 2016, ApJ, 820, L8

Maxted, P. F. L., Anderson, D. R., Collier Cameron, A., et al. 2011, PASP, 123, 547

Maxted, P. F. L., Serenelli, A. M., \& Southworth, J. 2015a, A\&A, 575, A36

Maxted, P. F. L., Serenelli, A. M., \& Southworth, J. 2015b, A\&A, 577, A90

Mayor, M., \& Queloz, D. 2012, New Astron. Rev., 56, 19

Mazeh, T., Holczer, T., \& Faigler, S. 2016, A\&A, 589, A75

McCormac, J., Pollacco, D., \& Skillen, I., et al. 2013, PASP, 125, 548

McCormac, J., Skillen, I., Pollacco, D., et al. 2014, MNRAS, 438, 3383

McKinney, W., et al. 2010, Proceedings of the 9th Python in Science Conference, 51

Miller, N., \& Fortney, J. J. 2011, ApJ, 736, L29

Morton, T. D. 2012, ApJ, 761, 6

Morton, T. D. 2015, Astrophysics Source Code Library [record ascl:1503.011]

Naoz, S., Farr, W. M., \& Rasio, F. A. 2012, ApJ, 754, L36

Nelson, B. E., Ford, E. B., \& Rasio, F. A. 2017, AJ, 154, 106

Parviainen, H., \& Aigrain, S. 2015, MNRAS, 453, 3821

Pepe, F., Mayor, M., Galland, F., et al. 2002, A\&A, 388, 632

Perez, F., \& Granger, B. E. 2011, Comput. Sci. Eng., 9, DOI: $10.1109 /$ MCSE.2007.53

Pollacco, D., Skillen, I., Collier Cameron, A., et al. 2006, PASP, 118, 1407

Pollacco, D., Skillen, I., Collier Cameron, A., et al. 2008, MNRAS, 385, 1576

Queloz, D., Mayor, M., Weber, L., et al. 2000, A\&A, 354, 99

Queloz, D., Henry, G. W., Sivan, J. P., et al. 2001, A\&A, 379, 279

Quintana, E. V., Jenkins, J. M., Clarke, B. D., et al. 2010, Proc. SPIE, 7740, $77401 X$

Rasio, F. A., \& Ford, E. B. 1996, Science, 274, 954

Rauer, H., Catala, C., Aerts, C., et al. 2014, Exp. Astron., 38, 249

Santerne, A., Díaz, R. F., Almenara, J.-M., et al. 2015, MNRAS, 451, 2337

Santos, N. C., Mayor, M., Naef, D., et al. 2002, A\&A, 392, 215

Schlaufman, K. C., \& Winn, J. N. 2016, ApJ, 825, 62

Schneider, J., Dedieu, C., Le Sidaner, P., Savalle, R., \& Zolotukhin, I. 2011, A\&A, 532, A79

Sestito, P., \& Randlich, S. 2005, A\&A, 442, 615

Skrutskie, M. F., Cutri, R. M., Stiening, R., et al. 2006, AJ, 131, 1163

Smalley, B. 2005, Mem. Soc. Astron. It. Suppl., 8, 130

Steele, I. A., et al. 2004, in SPIE Conf. Ser., 5489

Stetson, P. B. 1987, PASP, 99, 191

Szabó, G. M., \& Kiss, L. L. 2011, ApJ, 727, L44

Tamuz, O., Mazeh, T., \& Zucker, S. 2005, MNRAS, 356, 1466

Torres, G., Andersen, J., \& Giménez, A. 2010, A\&ARv, 18, 67

Torres, G., Fressin, F., Batalha, N. M., et al. 2011, ApJ, 727, 24

Van Der Walt, S., Colbert, S. C., \& Varoquaux, G. 2011, Comput. Sci. Eng., 13, 2

Ward, W. R. 1997, Icarus, 126, 261

Weiss, A., \& Schlattl, H. 2008, Ap\&SS, 316, 99

Welsh, W. F., Orosz, J. A., Carter, J. A., et al. 2012, Nature, 481, 475

Wheatley, P. J., et al. 2013, in Hot Planets and Cool Stars, EPJ Web Conf., 47, 13002

Wheatley, P. J., et al. 2014, in Exploring the formation and evolution of planetary systems, eds. Booth M., Matthews B. C., \& Graham J. R., Proc. IAU Symp., 299, 311

Zacharias, N., Finch, C. T., Girard, T. M., et al. 2013, AJ, 145, 44

Zeng, L., \& Sasselov, D. 2013, PASP, 125, 227 


\section{Appendix A: Additional tables}

Table A.4. System parameters from Bayesian MCMC analysis.

\begin{tabular}{|c|c|c|c|}
\hline & WASP-151b & WASP-153b & WASP-156b \\
\hline \multicolumn{4}{|l|}{ Planetary parameters } \\
\hline$R_{\mathrm{p}}\left[R_{\mathrm{Jup}}\right]($ adopted, from tr. + ev. track $)$ & $1.13_{-0.03}^{+0.03}$ & $1.55_{-0.08}^{+0.10}$ & $0.51_{-0.02}^{+0.02}$ \\
\hline$R_{\mathrm{p}}\left[R_{\mathrm{Jup}}\right]$ (from spec.) & $1.2_{-0.2}^{+0.2}$ & $1.1_{-0.2}^{+0.2}$ & $0.63_{-0.10}^{+0.10}$ \\
\hline$M_{\mathrm{p}}\left[M_{\mathrm{Jup}}\right]($ adopted, from tr. + ev. track $)$ & $0.31_{-0.03}^{+0.04}$ & $0.39_{-0.02}^{+0.02}$ & $0.128_{-0.009}^{+0.010}$ \\
\hline$M_{\mathrm{p}}\left[M_{\mathrm{Jup}}\right]$ (from spec.) & $0.33_{-0.03}^{+0.03}$ & $0.37_{-0.02}^{+0.02}$ & $0.13_{-0.01}^{+0.01}$ \\
\hline$\rho_{\mathrm{p}}\left[\rho_{\mathrm{Jup}}\right]($ adopted, from tr. + ev. track $)$ & $0.22_{-0.02}^{+0.03}$ & $0.11_{-0.02}^{+0.02}$ & $1.0_{-0.1}^{+0.1}$ \\
\hline$\rho_{\mathrm{p}}\left[\rho_{\mathrm{Jup}}\right]($ from spec. $)$ & $0.18_{-0.06}^{+0.11}$ & $0.3_{-0.1}^{+0.2}$ & $0.5_{-0.2}^{+0.3}$ \\
\hline$T_{\text {eq }}[\mathrm{K}]$ & $1.29 \times 10^{3+2 \times 10^{1}}$ & $1.70 \times 10^{3+4 \times 10^{1}}$ & $9.7 \times 10^{2+3 \times 10^{1}}$ \\
\hline$P^{\bullet}[$ days $]$ & $4.533471_{-4 \times 10^{-6}}^{+4 \times 10^{-6}}$ & $3.332609_{-2 \times 10^{-6}}^{+2 \times 10^{-6}}$ & $3.836169_{-3 \times 10^{-6}}^{+3 \times 10^{-6}}$ \\
\hline$t_{c} \cdot[\mathrm{BJD}-2400000]$ & $57741.0081_{-2 \times 10^{-4}}^{+1 \times 10^{-4}}$ & $53142.542_{-0.003}^{+0.003}$ & $54677.707_{-0.002}^{+0.002}$ \\
\hline$a[\mathrm{AU}]$ & $0.055_{-0.001}^{+0.001}$ & $0.048_{-0.001}^{+0.001}$ & $0.0453_{-0.0009}^{+0.0009}$ \\
\hline$e$ & $<0.003$ & $<0.009$ & $<0.007$ \\
\hline$\omega_{*}\left[{ }^{\circ}\right]$ & $-5 \times 10^{1+1.2 \times 10^{2}} \underset{-3 \times 10^{1}}{\stackrel{1}{1}}$ & unconstrained & unconstrained \\
\hline$i\left[^{\circ}\right]$ & $89.2_{-0.6}^{+0.6}$ & $84.1_{-0.7}^{+0.7}$ & $89.1_{-0.9}^{+0.6}$ \\
\hline$\sqrt{e} \cos \omega_{*}^{\bullet}$ & $0.000_{-9 \times 10^{-3}}^{+1.0 \times 10^{-2}}$ & $-0.00_{-0.05}^{+0.1}$ & $-0.01_{-0.10}^{+0.9}$ \\
\hline$\sqrt{e} \sin \omega_{*}^{\bullet}$ & $0.00_{-0.04}^{+0.03}$ & $-0.00_{-0.05}^{+0.05}$ & $0.00_{-0.05}^{+0.05}$ \\
\hline $\cos i^{\bullet}$ & $0.015_{-0.010}^{-0.04}$ & $0.10_{-0.01}^{+0.01}$ & $\begin{array}{r}-0.05 \\
0.02_{-0.01}^{+0.02}\end{array}$ \\
\hline$a / R_{*} \bullet$ & $10.34_{-0.19}^{+0.11}$ & $6.0_{-0.2}^{+0.3}$ & $12.8_{-0.7}^{+0.01}$ \\
\hline$R_{\mathrm{p}} / R_{*} \bullet[\%]$ & $10.11_{-0.03}^{+0.19}$ & $9.2_{-0.1}^{-0.2}$ & $6.85_{-0.08}^{+0.12}$ \\
\hline$\Delta F / F[\%]$ & $1.021_{-0.007}^{+0.01}$ & $0.85_{-0.02}^{+0.1}$ & $0.47_{-0.01}^{+0.02}$ \\
\hline$D 14[\mathrm{~h}]$ & $3.66_{-0.01}^{+0.02}$ & $3.84_{-0.05}^{-0.02}$ & $2.41_{-0.03}^{+0.01}$ \\
\hline$D 23[\mathrm{~h}]$ & $2.97_{-0.02}^{+0.01}$ & $2.83_{-0.06}^{+0.03}$ & $2.08_{-0.03}^{+0.03}$ \\
\hline$K^{\bullet}\left[\mathrm{m} \mathrm{s}^{-1}\right]$ & $37_{-3}^{+4}$ & $44_{-2}^{+2}$ & $19_{-1}^{+1}$ \\
\hline$\tau_{\text {circ }}[\mathrm{Gyr}]$ & $0.03_{-0.003}^{+0.004}$ & $0.0022_{-0.0006}^{+0.0006}$ & $0.26_{-0.05}^{+0.06}$ \\
\hline$H[\mathrm{~km}]($ adopted, from tr. + ev. track $)$ & $8.0 \times 10_{-8 \times 10^{1}}^{2+9 \times 10^{1}}$ & $1.6 \times 10_{-2 \times 10^{2}}^{3+2 \times 10^{2}}$ & $3.0 \times 10^{2+4 \times 10^{1}}$ \\
\hline$H[\mathrm{~km}]$ (from spec.) & $9.0 \times 10^{2+3 \times 10^{2}}$ & $8 \times 10^{2+3 \times 10^{2}}$ & $5 \times 10^{2+2 \times 10^{2}}$ \\
\hline$F_{i}\left[F_{i, \oplus}\right]($ adopted, from tr. + ev. track $)$ & $4.6 \times 10_{-2 \times 10^{1}}^{2+2 \times 10^{1}}$ & $1.4 \times 10^{3+2 \times 10^{2}}$ & $1.5 \times 10^{2+1 \times 10^{1}}$ \\
\hline$F_{i}\left[F_{i, \oplus}\right]($ from spec. $)$ & $5 \times 10^{2}+2 \times 10^{2}$ & $7 \times 10^{2+3 \times 10^{2}}+2 \times 10^{2}$ & $2 \times 10^{2+8 \times 10^{1}}$ \\
\hline \multicolumn{4}{|l|}{ Stellar parameters } \\
\hline RA [hours:minutes:s] & $23: 16: 15.22$ & $18: 37: 02.97$ & 02:11:07.61 \\
\hline Dec [degrees minutes s] & 001824.5 & 400107.4 & 022504.8 \\
\hline Sp. Type (spec.) & G1 & G0 & $\mathrm{K} 3$ \\
\hline
\end{tabular}

Notes. (spec.) indicates that the estimate has been performed using the spectroscopic data only (Sect. 3.1). (tr.) indicates that the estimate has been performed using transit and RV analysis only (Sect. 3.2.1). (spec., tr.) indicates that the estimate has been performed using both transit and spectroscopic data (Sect. 3.2.1). $M_{*}$ and $R_{*}$ (spec.) estimates are performed using the spectroscopic $T_{\text {eff }}, \log g$, [Fe/H] and the Torres et al. (2010) calibration. $M_{*}$ and $R_{*}\left(t r .+e v\right.$. track) are provided by bagemass using $\rho_{*}(t r), T_{\text {eff }}$ and $[\mathrm{Fe} / \mathrm{H}]$ (spec.) (Sect. 3.2.2). (from spec.) indicates that the estimate has been performed using $M_{*}$ and $R_{*}$ (spec.) estimates. (adopted, from tr. + ev. track) indicates that the estimate has been performed using $M_{*}$ and $R_{*}(t r .+e v$. track) estimates and that we adopted those values as final values for the system. We believe that those values are more accurate than the one provided the spectroscopic parameters and the Torres et al. (2010) calibration. Spectral Types are estimated from $T_{\text {eff }}$ using the table in Gray (2008). Abundances are relative to the solar values obtained by Asplund et al. (2009). $\bullet$ indicates that the parameter is a jumping parameter in the MCMC analysis. For more details of the meaning of the notations used for the parameters name, see Sect. 3.2. 
Table A.4. continued.

\begin{tabular}{|c|c|c|c|}
\hline & WASP-151b & WASP-153b & WASP-156b \\
\hline$V$ mag & 12.9 & 12.8 & 11.6 \\
\hline$J$ mag & 11.5 & 11.4 & 9.9 \\
\hline$V-K$ & 1.7 & 1.7 & 2.2 \\
\hline$M_{*}\left[M_{\odot}\right]($ adopted, tr. + ev. track. $)$ & $1.077 \pm 0.081$ & $1.336 \pm 0.086$ & $0.842 \pm 0.052$ \\
\hline$M_{*}\left[M_{\odot}\right]($ spec. $)$ & $1.14 \pm 0.09$ & $1.20 \pm 0.09$ & $0.87 \pm 0.07$ \\
\hline$R_{*}\left[R_{\odot}\right]($ adopted, tr. + ev. track. $)$ & $1.14_{-0.03}^{+0.03}$ & $1.73_{-0.09}^{+0.10}$ & $0.76_{-0.03}^{+0.03}$ \\
\hline$R_{*}\left[R_{\odot}\right]($ spec. $)$ & $1.24 \pm 0.18$ & $1.18 \pm 0.20$ & $0.95 \pm 0.15$ \\
\hline Age [Gyr] (adopted, Isochrone) & $5.1_{-1.3}^{+1.3}$ & $4.0_{-0.8}^{+0.8}$ & $6.4_{-4.0}^{+4.0}$ \\
\hline Age [Gyr] (Gyrochronology) & $1.80_{-1.00}^{+2.03}$ & $1.21_{-0.60}^{+1.19}$ & $0.58_{-0.31}^{+0.51}$ \\
\hline$\rho_{*}\left[\rho_{\odot}\right](t r)$. & $0.72_{-0.04}^{+0.02}$ & $0.26_{-0.03}^{+0.04}$ & $1.9_{-0.3}^{+0.1}$ \\
\hline$T_{\mathrm{eff}}[\mathrm{K}]($ spec.) & $5871 \pm 57$ & $5914 \pm 64$ & $4910 \pm 61$ \\
\hline $\log g$ (spec.) & $4.30 \pm 0.11$ & $4.36 \pm 0.13$ & $4.40 \pm 0.12$ \\
\hline $\log g$ (adopted, tr.) & $4.35_{-0.03}^{+0.02}$ & $4.10_{-0.06}^{+0.06}$ & $4.60_{-0.07}^{+0.04}$ \\
\hline$[\mathrm{Fe} / \mathrm{H}][\mathrm{dex}]($ spec.) & $0.10 \pm 0.10$ & $0.34 \pm 0.11$ & $0.24 \pm 0.12$ \\
\hline$v \sin i_{*}\left[\mathrm{~km} \mathrm{~s}^{-1}\right]$ (spec.) & $4.25 \pm 0.90$ & $5.19 \pm 0.95$ & $3.80 \pm 0.91$ \\
\hline$v_{\mathrm{mac}}\left[\mathrm{km} \mathrm{s}^{-1}\right]$ (spec.) & $3.73 \pm 0.73$ & $3.73 \pm 0.73$ & $2.77 \pm 0.73$ \\
\hline$\xi_{\mathrm{t}}\left[\mathrm{km} \mathrm{s}^{-1}\right]($ spec. $)$ & $0.32 \pm 0.10$ & $0.59 \pm 0.06$ & \\
\hline $\log A(\mathrm{Li})$ (spec.) & $1.73 \pm 0.05$ & $2.77 \pm 0.05$ & $<0.19 \pm 0.08$ \\
\hline Distance [pc] (spec.) & $480 \pm 75$ & $430 \pm 35$ & $140 \pm 25 \mathrm{pc}$ \\
\hline$v 0_{\text {SOPHIE }} \cdot\left[\mathrm{km} \mathrm{s}^{-1}\right](t r)$. & $-12.369_{-0.002}^{+0.002}$ & $-29.004_{-0.001}^{+0.002}$ & $9.5891_{-9 \times 10^{-4}}^{+9 \times 10^{-4}}$ \\
\hline$u_{\text {Johson } R^{\bullet}}($ spec., tr. $)$ & $0.478_{-0.002}^{+0.002}$ & $0.486_{-0.002}^{+0.002}$ & $0.591_{-0.003}^{+0.003}$ \\
\hline$v_{\text {Johson } R^{\bullet}}($ spec., tr. $)$ & $0.129_{-0.005}^{+0.005}$ & $0.126_{-0.005}^{+0.005}$ & $0.082_{-0.008}^{+0.008}$ \\
\hline$u_{\text {Sloan } z} \bullet($ spec., tr. $)$ & $0.341_{-0.001}^{+0.001}$ & & \\
\hline$v_{\text {Sloan } z} \bullet($ spec., tr. $)$ & $0.127_{-0.004}^{-0.001}$ & & \\
\hline$u_{\mathrm{Kp}} \bullet($ spec., tr. $)$ & $0.549_{-0.003}^{+0.004}$ & & \\
\hline$v_{\mathrm{Kp}} \bullet($ spec., tr. $)$ & $0.114_{-0.005}^{+0.005}$ & & \\
\hline$u_{\mathrm{NGTS}}{ }^{\bullet}($ spec., $t r)$. & $0.487_{-0.002}^{+0.002}$ & & \\
\hline$v_{\mathrm{NGTS}} \bullet($ spec., $t r)$. & $0.131_{-0.005}^{+0.004}$ & & \\
\hline$u_{\mathrm{V}+\mathrm{R}} \bullet($ spec., $t r)$. & & $0.549_{-0.002}^{+0.002}$ & \\
\hline$v_{\mathrm{V}+\mathrm{R}} \bullet($ spec., tr. $)$ & & $0.118_{-0.006}^{+0.006}$ & \\
\hline$u_{\text {Johson } I} I^{\bullet}($ spec., tr. $)$ & & & $0.461_{-0.002}^{+0.002}$ \\
\hline$v_{\mathrm{Johson} I} \bullet^{\bullet}($ spec., $t r)$. & & & $0.0995_{-0.0074}^{+0.0066}$ \\
\hline$u_{\mathrm{Gunn} r} \bullet($ spec., tr. $)$ & & & $0.461_{-0.002}^{+0.002}$ \\
\hline$v_{\mathrm{Gunn} r} r^{(\text {spec., } t r .)}$ & & & $0.103_{-0.007}^{+0.007}$ \\
\hline \multicolumn{4}{|l|}{ Parameters of instruments } \\
\hline$\Delta \mathrm{RV}_{\mathrm{CORALIE} / \mathrm{SOPHIE}}{ }^{\bullet}\left[\mathrm{km} \mathrm{s}^{-1}\right]$ & $0.055_{-0.009}^{+0.008}$ & & $0.043_{-0.002}^{+0.002}$ \\
\hline $\ln f_{\sigma \mathrm{SOPHIE}}{ }^{\bullet}$ & $0.03_{-0.05}^{+0.04}$ & $0.04_{-0.04}^{+0.04}$ & $0.08_{-0.04}^{+0.04}$ \\
\hline $\ln f_{\sigma \text { CORALIE }}{ }^{\bullet}$ & $-0.01_{-0.06}^{+0.06}$ & & $-0.01_{-0.05}^{+0.05}$ \\
\hline $\ln f_{\sigma \mathrm{K} 2} \bullet$ & $1.71_{-0.02}^{+0.02}$ & & \\
\hline $\ln f_{\sigma \text { Euler } \mathrm{Cam}}^{\bullet}$ & $0.24_{-0.03}^{+0.03}$ & & $0.43_{-0.03}^{+0.03}$ \\
\hline $\ln f_{\sigma \mathrm{TRAPPIST}}{ }^{\bullet}$ & $0.01_{-0.02}^{+0.03}$ & & \\
\hline
\end{tabular}


O. Demangeon et al.: Discovery of WASP-151b, WASP-153b and WASP-156b

Table A.4. continued.

\begin{tabular}{|c|c|c|c|}
\hline & WASP-151b & WASP-153b & WASP-156b \\
\hline $\ln f_{\sigma \mathrm{WASP}}{ }^{\bullet}$ & $0.112_{-0.009}^{+0.008}$ & $-0.056_{-0.008}^{+0.008}$ & $0.31_{-0.01}^{+0.01}$ \\
\hline $\ln f_{\sigma \text { Liverpool }}{ }^{\bullet}$ & & $-1.601_{-0.006}^{+0.006}$ & \\
\hline $\ln f_{\sigma \mathrm{RISE} 2}^{\bullet}$ & & $-0.81_{-0.02}^{+0.02}$ & \\
\hline $\ln f_{\sigma \text { NITES }}^{\bullet}$ & & & $0.39_{-0.02}^{+0.03}$ \\
\hline $\ln f_{\sigma \mathrm{IAC} 80^{\bullet}}$ & $\begin{array}{l}-0.32_{-0.05}^{+0.04} \\
-0.26_{-0.05}^{+0.05} \\
-0.14_{-0.05}^{+0.05}\end{array}$ & & \\
\hline$\Delta F_{\mathrm{OOT}, \mathrm{IAC} 80}$ & $\begin{array}{c}0.0042_{-4 \times 10^{-4}}^{+4 \times 10^{-4}} \\
-7 \times 10^{-4}+6 \times 10^{-4} \\
-6 \times 10^{-4} \\
-1 \times 10^{-4}+8 \times 10^{-4} \\
-8 \times 10^{-4}\end{array}$ & & \\
\hline$\Delta F_{\text {OOT,IAC } 80}^{\prime} \cdot\left[\right.$ day $\left.^{-1}\right]$ & $\begin{array}{c}-0.024_{-0.003}^{+0.004} \\
-0.008_{-0.007}^{+0.007} \\
0.02_{-0.02}^{+0.02}\end{array}$ & & \\
\hline$\Delta F_{\text {OOT,TRAPPIST }}{ }^{\bullet}$ & $-2 \times 10^{-6}+3 \times 10^{-4}$ & & \\
\hline$\Delta F_{\text {OOT,TRAPPIST }}^{\prime} \cdot\left[\right.$ day $\left.^{-1}\right]$ & $0.008_{-0.004}^{+0.004}$ & & \\
\hline$\Delta F_{\mathrm{OOT}, \text { Euler } \mathrm{Cam}} \bullet$ & & & $7 \times 10^{-6+1 \times 10^{-4}}+1 \times 10^{-4}$ \\
\hline 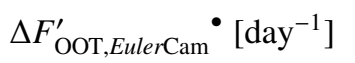 & & & $-5 \times 10^{-4}+9 \times 10^{-4}$ \\
\hline$\Delta F_{\text {OОТ,EulerCam }}^{\prime \prime} \cdot\left[\mathrm{day}^{-2}\right]$ & & & $-0.001_{-0.001}^{+0.001}$ \\
\hline
\end{tabular}


Table A.5. Prior functions for each free parameters.

\begin{tabular}{|c|c|c|c|}
\hline Parameters & WASP-151b & WASP-153b & WASP-156b \\
\hline$P$ [days] & $\mathcal{N}(4.5334,0.003)$ & $\mathcal{N}(3.333,0.001)$ & $\mathcal{N}\left(3.83616,3 \times 10^{5}\right)$ \\
\hline$t_{c}[\mathrm{BJD}-2400000]$ & $\mathcal{N}(57741.0,0.1)$ & $\mathcal{N}(53142.5,0.1)[\mu-2 \sigma, \mu+2 \sigma]$ & $\mathcal{N}(54677.71,0.01)$ \\
\hline$\sqrt{e} \cos \omega_{*}$ & & $\mathcal{N}(0,0.05)[0,1 / \sqrt{2}]$ & - \\
\hline$\sqrt{e} \sin \omega_{*}$ & & $\mathcal{N}(0,0.05)[0,1 / \sqrt{2}]$ & \\
\hline $\cos i$ & & $-\mathcal{N}(0,0.1)[0 ., 1]$. & \\
\hline$a / R_{*}$ & $\mathcal{N}(8.67,1)[1 ., 30]$ & $\mathcal{N}(8.4,1)[1,50]$ & $\mathcal{U}(1 ., 30)$ \\
\hline$R_{\mathrm{p}} / R_{*}[\%]$ & & $\mathcal{U}(1,20)$ & \\
\hline$K\left[\mathrm{~m} \mathrm{~s}^{-1}\right]$ & & $\mathcal{U}(0,1)$ & \\
\hline$v 0_{\text {SOPHIE }}\left[\mathrm{km} \mathrm{s}^{-1}\right]$ & $\mathcal{N}(-12.4,0.02)$ & $\mathcal{N}(-29,0.1)$ & $\mathcal{N}(9.58,0.01)$ \\
\hline$u_{\text {Johson } R}$ & $\mathcal{N}(0.4781,0.0022)$ & $\mathcal{N}(0.4859,0.0020)$ & $\mathcal{N}(0.5911,0.0032)$ \\
\hline$v_{\text {Johson } R}$ & $\mathcal{N}(0.1304,0.0055)$ & $\mathcal{N}(0.1258,0.0053)$ & $\mathcal{N}(0.0805,0.0082)$ \\
\hline$u_{\text {Sloan } z}$ & $\mathcal{N}(0.3412,0.0013)$ & & \\
\hline$v_{\text {Sloan } z}$ & $\mathcal{N}(0.1269,0.0039)$ & & \\
\hline$u_{\mathrm{Kp}}$ & $\mathcal{N}(0.5501,0.0025)$ & & \\
\hline$v_{\mathrm{Kp}}$ & $\mathcal{N}(0.1191,0.0054)$ & & \\
\hline$u_{\text {NGTS }}$ & $\mathcal{N}(0.4861,0.0021)$ & & \\
\hline$v_{\text {NGTS }}$ & $\mathcal{N}(0.1286,0.0052)$ & & \\
\hline$u_{\mathrm{Johson} I}$ & & & $\mathcal{N}(0.4608,0.0024)$ \\
\hline$v_{\text {Johson } I}$ & & & $\mathcal{N}(0.1013,0.0070)$ \\
\hline$u_{\mathrm{Gunn} r} r$ & & & $\mathcal{N}(0.4608,0.0024)$ \\
\hline$v_{\mathrm{Gunn} r}$ & & & $\mathcal{N}(0.0812,0.0085)$ \\
\hline$\Delta \mathrm{RV}_{\text {CORALIE/SOPHIE }}\left[\mathrm{km} \mathrm{s}^{-1}\right]$ & $\mathcal{N}(0.05,0.01)$ & & $\mathcal{N}(0.05,0.005)$ \\
\hline $\ln f_{\sigma \text { SOPHIE }}$ & & $-\mathcal{N}(0,0.05)-$ & \\
\hline $\ln f_{\sigma \text { CORALIE }}$ & $\mathcal{N}(0,0.05)$ & & $\mathcal{N}(0,0.05)$ \\
\hline $\ln f_{\sigma \mathrm{WASP}}$ & & $-\mathcal{N}(0,0.05)-$ & \\
\hline $\ln f_{\sigma \mathrm{K} 2}$ & $\mathcal{N}(0,0.05)$ & & \\
\hline $\ln f_{\sigma E u l e r \mathrm{Cam}}$ & $\mathcal{N}(0,0.05)$ & & $\mathcal{N}(0,0.05)$ \\
\hline $\ln f_{\sigma \text { TRAPPIST }}$ & $\mathcal{N}(0,0.05)$ & & \\
\hline $\ln f_{\sigma \text { Liverpool }}$ & & $\mathcal{N}(0,0.05)$ & \\
\hline $\ln f_{\sigma \mathrm{RISE} 2}$ & & $\mathcal{N}(0,0.05)$ & \\
\hline $\ln f_{\sigma \text { NITES }}$ & & & $\mathcal{N}(0,0.05)$ \\
\hline $\ln f_{\sigma \mathrm{IAC} 80}$ & $\mathcal{N}(0,0.05)$ & & \\
\hline \multirow[t]{3}{*}{$\Delta F_{\text {OOT,IAC } 80}$} & $\mathcal{N}(0.005,0.01)$ & & \\
\hline & $\mathcal{N}(0.0,0.01)$ & & \\
\hline & $\mathcal{N}(0.0,0.01)$ & & \\
\hline \multirow[t]{3}{*}{$\Delta F_{\text {OOT,IAC80 }}^{\prime}\left[\right.$ day $\left.^{-1}\right]$} & $\mathcal{N}(0.0,0.33)$ & & \\
\hline & $\mathcal{N}(0.0,0.1)$ & & \\
\hline & $\mathcal{N}(0.0,0.1)$ & & \\
\hline$\Delta F_{\text {OOT,TRAPPIST }}$ & $\mathcal{N}(0.0,0.001)$ & & \\
\hline$\Delta F_{\text {OOT.TRAPPIST }}^{\prime}\left[\mathrm{day}^{-1}\right]$ & $\mathcal{N}(0.0,0.005)$ & & \\
\hline$\Delta F_{\text {OOT,EulerCam }}$ & & & $\mathcal{N}(-0.00036,0.001)$ \\
\hline$\Delta F_{\text {OOT,EulerCam }}^{\prime}\left[\right.$ day $\left.^{-1}\right]$ & & & $\mathcal{N}(0.0012,0.001)$ \\
\hline$\Delta F_{\text {OOT,EulerCam }}^{\prime \prime}\left[\right.$ day $\left.^{-2}\right]$ & & & $\mathcal{N}(-0.001,0.001)$ \\
\hline
\end{tabular}

Notes. $\mathcal{N}(\mu, \sigma)$ designate normal distributions of mean $\mu$ and standard deviation $\sigma . \mathcal{N}(\mu, \sigma)[\min , \max ]$ designate truncated normal distributions with min and max as minimum and maximum value. $\mathcal{U}(\min , \max )$ designate uniform distributions with min and max as minimum and maximum value. - distribution - indicates that the same prior distribution has been used for the analysis of the three systems. 
O. Demangeon et al.: Discovery of WASP-151b, WASP-153b and WASP-156b

Table A.6. Complete bagemass output table giving the Bayesian mass and age estimates for WASP-151, WASP-153, and WASP-156.

\begin{tabular}{|c|c|c|c|c|c|c|c|c|c|c|c|}
\hline Star & $\tau_{\text {iso,b }}[\mathrm{Gyr}]$ & $M_{\mathrm{b}}\left[M_{\odot}\right]$ & {$[\mathrm{Fe} / \mathrm{H}]_{\mathrm{i}, \mathrm{b}}$} & $\chi^{2}$ & $\left\langle\tau_{\text {iso }}\right\rangle[\mathrm{Gyr}]$ & $\left\langle M_{\star}\right\rangle\left[M_{\odot}\right]$ & $p_{\mathrm{MS}}$ & $\sigma_{\tau, Y}$ & $\sigma_{\tau, \alpha}$ & $\sigma_{M, Y}$ & $\sigma_{M, \alpha}$ \\
\hline WASP-151 & 5.0 & 1.07 & +0.162 & 0.002 & $5.13 \pm 1.33$ & $1.077 \pm 0.048$ & 1.00 & -0.42 & 2.59 & 0.044 & -0.049 \\
\hline WASP-153 & 3.8 & 1.35 & +0.339 & 0.015 & $4.00 \pm 0.77$ & $1.336 \pm 0.065$ & 0.87 & -0.08 & 0.79 & 0.052 & -0.021 \\
\hline WASP-156 & 0.5 & 0.87 & +0.255 & 0.02 & $6.50 \pm 4.03$ & $0.842 \pm 0.036$ & 1.00 & -0.68 & 1.37 & 0.034 & -0.017 \\
\hline
\end{tabular}

Notes. Columns 2-4 give the maximum-likelihood estimates of the age, mass, and initial metallicity, respectively. Column 5 is the chi-squared statistic of the fit for the parameter values in Cols. 2-4. Columns 6 and 7 give the mean and standard deviation of their marginalized posterior distributions. Column $8\left(p_{\mathrm{MS}}\right)$ is the probability that the star is still on the main sequence. The systematic errors on the mass and age due to uncertainties in the mixing length and helium abundance are given in Cols. 9 to 12. For more details see Sect. 3.2.2 and Maxted et al. (2015a). 